\title{
Fas/FasL mediates NF-kBp65/PUMA-modulated hepatocytes apoptosis via autophagy to drive liver fibrosis
}

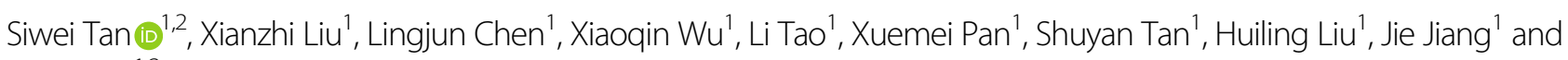
Bin Wu $\mathbb{B}^{1,2}$

\begin{abstract}
Fas/Fas ligand (FasL)-mediated cell apoptosis involves a variety of physiological and pathological processes including chronic hepatic diseases, and hepatocytes apoptosis contributes to the development of liver fibrosis following various causes. However, the mechanism of the Fas/FasL signaling and hepatocytes apoptosis in liver fibrogenesis remains unclear. The Fas/FasL signaling and hepatocytes apoptosis in liver samples from both human sections and mouse models were investigated. NF-KBp65 wild-type mice ( $p 65^{f / f}$ ), hepatocytes specific NF-KBp65 deletion mice ( $p 65 \Delta$ hepa), p53-upregulated modulator of apoptosis (PUMA) wild-type (PUMA-WT) and PUMA knockout (PUMA-KO) littermate models, and primary hepatic stellate cells (HSCs) were also used. The mechanism underlying Fas/FasL-regulated hepatocytes apoptosis to drive HSCs activation in fibrosis was further analyzed. We found Fas/FasL promoted PUMAmediated hepatocytes apoptosis via regulating autophagy signaling and NF-kBp65 phosphorylation, while inhibition of autophagy or PUMA deficiency attenuated Fas/FasL-modulated hepatocytes apoptosis and liver fibrosis.

Furthermore, NF-KBp65 in hepatocytes repressed PUMA-mediated hepatocytes apoptosis via regulating the Bcl-2 family, while NF-KBp65 deficiency in hepatocytes promoted PUMA-mediated hepatocytes apoptosis and enhanced apoptosis-linked inflammatory response, which contributed to the activation of HSCs and liver fibrogenesis. These results suggest that Fas/FasL contributes to NF-KBp65/PUMA-modulated hepatocytes apoptosis via autophagy to enhance liver fibrogenesis, and this network could be a potential therapeutic target for liver fibrosis.
\end{abstract}

\section{Introduction}

Liver fibrosis represents one of the major consequences of morbidity and mortality worldwide ${ }^{1,2}$, and the activation of hepatic stellate cells (HSCs), which is regulated by multiple cell populations or soluble mediators, is the major source of extracellular matrix substances $(\mathrm{ECM})^{3-5}$. Hepatocytes are the major parenchymal cells of the liver and essential for maintaining the function and

\footnotetext{
Correspondence: Siwei Tan (xiaodatou520@sina.com) or Bin Wu (wubin6@mail. sysu.edu.cn)

${ }^{1}$ Department of Gastroenterology, the Third Affiliated Hospital of Sun Yat-Sen University, Guangzhou, Guangdong Province 510630, China

${ }^{2}$ Guangdong Provincial Key Laboratory of Liver Disease Research, Guangzhou, Guangdong Province 510630, China

These authors contributed equally: Siwei Tan, Xianzhi Liu

Edited by B. Zhivotovsky
}

organization of the liver ${ }^{6}$. It's widely accepted that the progression of hepatic fibrosis is associated with considerable injury and loss of hepatocytes, which may present as a main inflammatory stimulus for HSCs activation $^{7,8}$. The apoptotic cells could release the nucleotides ATP and UTP, which bind to purinergic receptors (especially the $\mathrm{P}_{2} \mathrm{Y}_{2}$ receptor) on macrophages and HSCs, leading to their activation ${ }^{9,10}$. As a classic apoptosis modulator, Fas, following Fas ligand (FasL) engagement, leads to the recruitment of Fas-associated proteins having death domains and the initiators into a death-inducing signaling complex (DISC) to cause apoptosis $^{11-13}$, and the Fas/FasL has been demonstrated to participate in the pathological processes of hepatic

\section{(c) The Author(s) 2021}

(c) (i) Open Access This article is licensed under a Creative Commons Attribution 4.0 International License, which permits use, sharing, adaptation, distribution and reproduction c. in any medium or format, as long as you give appropriate credit to the original author(s) and the source, provide a link to the Creative Commons license, and indicate if changes were made. The images or other third party material in this article are included in the article's Creative Commons license, unless indicated otherwise in a credit line to the material. If material is not included in the article's Creative Commons license and your intended use is not permitted by statutory regulation or exceeds the permitted use, you will need to obtain permission directly from the copyright holder. To view a copy of this license, visit http://creativecommons.org/licenses/by/4.0/. 
fibrosis/cirrhosis, acute/chronic hepatitis and hepatocarcinoma ${ }^{14-16}$.

Autophagy is an evolutionarily conserved and catabolic process that targets cytosolic material, organelles and long-lived proteins to lysosomes to be degraded for survival, development, differentiation, and homeostasis ${ }^{17-19}$. Hepatocytes have been revealed to own a high level of autophagic flux because of their increased abundance of lysosomes and lysosomal enzymes, and the enhanced autophagy could modulate the progression to hepatocytes death $^{20}$, and our previous study has suggested autophagy was required for liver fibrogenesis ${ }^{21}$. Nuclear factor- $\mathrm{kB}$ $(\mathrm{NF}-\mathrm{kB})$, as a ubiquitous and inducible transcription factor responsible for mediating the expression of a large number of genes involved in differentiation, apoptosis, and proliferation ${ }^{22}$, and NF- $\mathrm{kBp} 65$, as the main functional element, involves in various physiological and pathological events and influences the survival of hepatocytes and activation of $\mathrm{HSCs}^{23}$. Activation of NF-kB in nonparenchymal cells promotes inflammation, fibrosis, and hepatocarcinogenesis in the liver, whereas suppression of $\mathrm{NF}-\kappa \mathrm{B}$ in parenchymal cells enhances hepatocarcinogenesis in some cases and retards hepatocarcinogenesis in others $^{10,24}$. The activation of NF-kB in HSCs appears to promote hepatic fibrosis via modulating fibrogenic effects and anti-apoptotic effects ${ }^{9}$. While decreased or absent NF- $\kappa B$ activity in hepatocytes might lead to subsequent fibrosis by regulating hepatocytes injury and the primary trigger of fibrogenic responses in liver ${ }^{23-26}$.

Considerable death and loss of hepatocytes induced by different etiologies are always associated with the initiation and progression of hepatic fibrosis ${ }^{1,27}$. p53upregulated modulator of apoptosis (PUMA), is one of the most potent mediators of apoptosis induced by various stimuli ${ }^{28}$. PUMA deficiency could block cell apoptotic responses to p53 activation, DNA-damaging agents, and hypoxia in hepatocytes ${ }^{29}$. In response to multiple insults, PUMA functions through other Bcl-2 family members, including Bcl-2, Mcl-1, and Bcl-xL, to induce mitochondrial dysfunction and caspases activation ${ }^{30,31}$. PUMA-mediated apoptotic response in hepatocytes is a direct cause of compensatory proliferation and carcinogenesis in the liver ${ }^{32}$. Mice deficient in $N F-\kappa B$ signaling or the anti-apoptotic Bcl-2 family members developed spontaneous hepatocellular carcinoma secondary to hepatocytes death ${ }^{33}$, and PUMA has been demonstrated to be a target of NF-kBp65 and a critical mediator of TNF- $\alpha$-induced hepatocytes apoptosis ${ }^{34}$. However, the role of NF-кBp65 in PUMA-regulated hepatocytes apoptosis in liver fibrogenesis remains unknown.

In the current study, we found that Fas/FasL promoted PUMA-mediated hepatocytes apoptosis via autophagy and NF-kBp65 signaling, while inhibition of autophagy or PUMA deficiency attenuated Fas/FasL-modulated hepatocytes apoptosis and liver fibrosis. Furthermore, hepatocytes deletion of NF- $\mathrm{KBp} 65$ promoted PUMAmediated hepatocytes apoptosis via regulating the $\mathrm{Bcl}-2$ family, and the enhanced hepatocytes apoptosis linked inflammatory action to drive HSCs activation and liver fibrogenesis. Thus, we suggest that Fas/FasL contributes to NF-kBp65/PUMA-regulated hepatocytes apoptosis via autophagy to enhance liver fibrogenesis.

\section{Results}

Fas/FasL-mediated apoptosis involved in liver fibrogenesis

To evaluate the role of Fas/FasL in liver fibrosis, liver specimens from healthy volunteers and liver fibrosis patients were analyzed. Histological staining presented a loss of preserved architecture and excess deposition of ECM, associated with obvious hepatic apoptosis and upregulated Fas/FasL expression, were observed in the liver fibrotic tissues compared with the normal samples (Fig. 1a, b). Western blotting also confirmed a similar condition (Fig. 1c, d). By using mouse model, we found that $\mathrm{CCl}_{4}$ injection-induced prominent liver fibrosis in mice as shown by $\alpha$-SMA and COL-I staining, which was accompanied with obvious apoptosis and Fas/FasL upregulation (Fig. 1a, b), and western blotting further represented that the levels of Fas, FasL, $\alpha$-SMA, COL-I, COL$\mathrm{IV}$, and cleaved caspase- 3 were enhanced in the fibrotic sections (Fig. 1c, d). These results indicated that Fas/FasL involved in hepatic apoptosis in liver fibrogenesis.

\section{Autophagy participated in Fas/FasL-mediated hepatic apoptosis in liver fibrosis}

Our preceding data has testified that autophagy was required for liver fibrosis ${ }^{21}$. In the current study, we found that the autophagic element BECN1 was enhanced in liver fibrosis, which was associated with the upregulated $\alpha$-SMA and COL-I expressions and apoptotic signaling (Fig. 2a), and ultrastructural analysis showed the typical autophagosomes in the hepatocytes of mouse and human liver fibrotic sections but scarce in the normal tissues (Fig. 2b). We pretreated mice with autophagic inhibitor 3-methyladenine (3-MA) and found 3-MA treatment alleviated the degree of apoptosis and liver fibrosis in mice, and did not affect the levels of Fas and FasL (Fig. 2a, c and Supplementary Fig. 1a). To sum up, these data suggested that autophagy participated in Fas/ FasL-mediated hepatic apoptosis in liver fibrosis.

\section{PUMA responded to Fas/FasL/autophagy-regulated hepatocytes apoptosis and HSCs activation during liver fibrosis}

PUMA has been demonstrated to be a target of Fas/ FasL signaling and a critical mediator of apoptosis in our previous study ${ }^{35}$. By analyzing the mouse models, we found that, accompanying by the loss of preserved 
$\mathbf{a}$
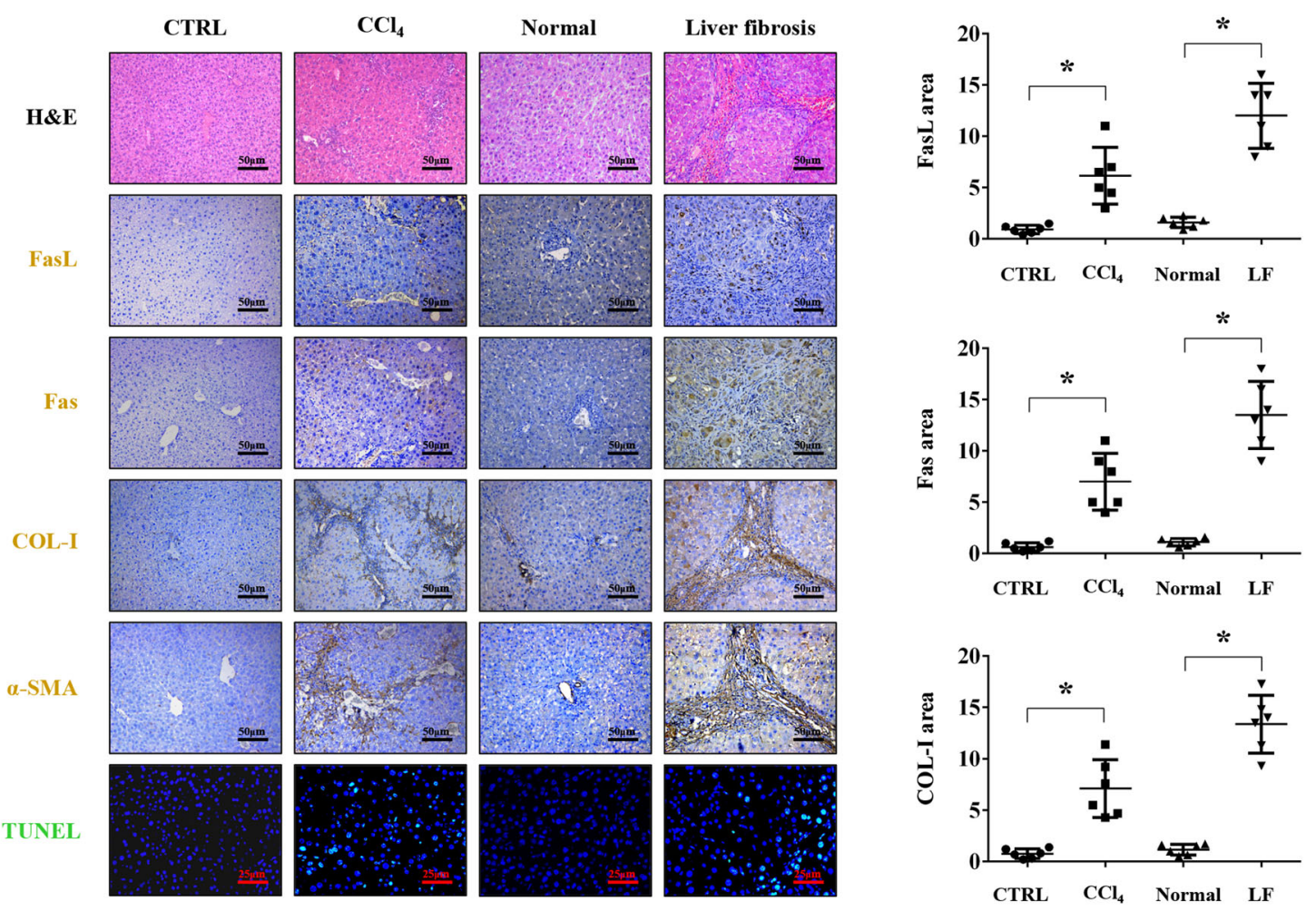

b

c
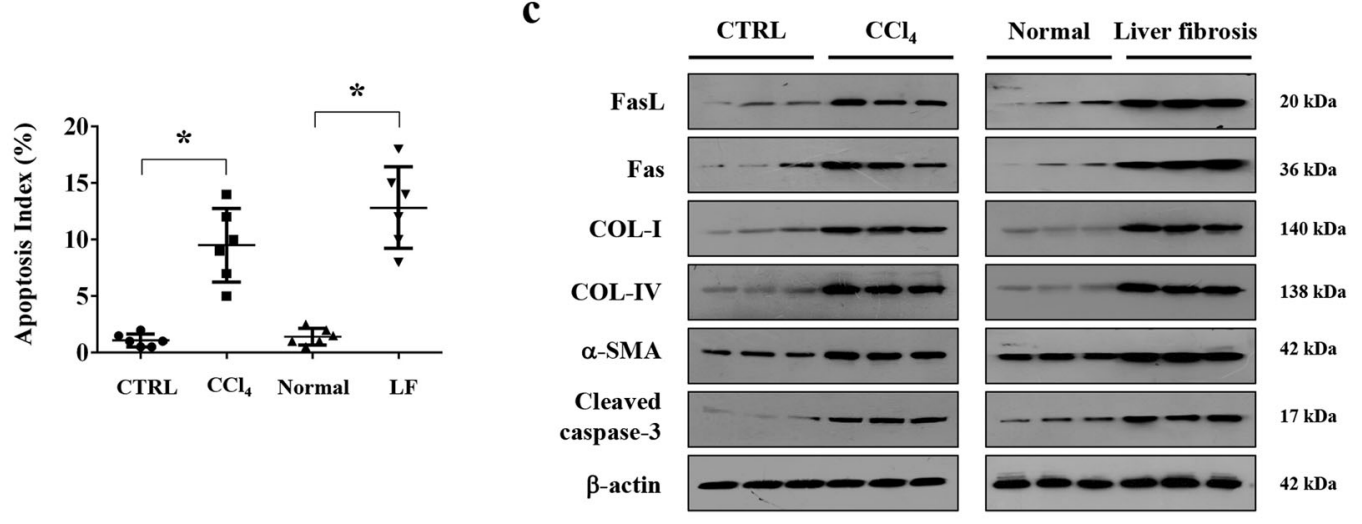

d
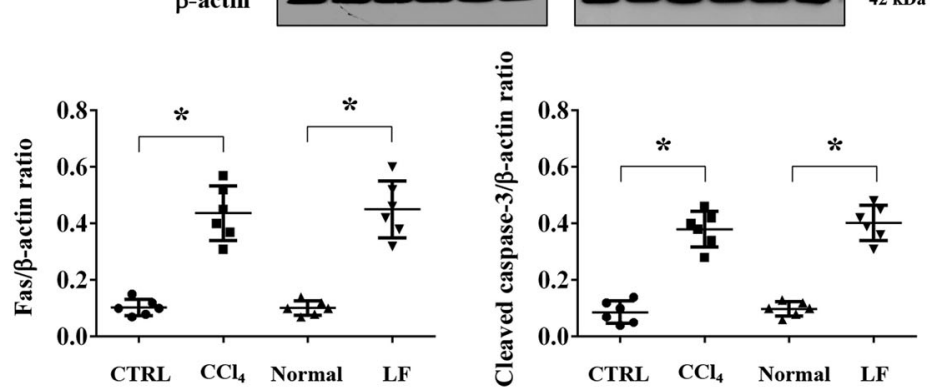

Fig. 1 Fas/FasL-mediated apoptosis involved in liver fibrogenesis. a H\&E staining, Fas, FasL, collagen-I (COL-I), a-SMA immunohistochemical staining (brown), and TUNEL staining (green) in the related liver tissues were presented. FasL, Fas, and COL-I area from the histological staining were also determined. Values are presented as mean \pm SEM. ${ }^{*} P<0.05, n=6$ per group. CTRL, the control mice (olive oil-treated mice); $C \mathrm{CCl}, 20 \%$ carbon tetrachloride-induced mouse fibrosis; Normal, healthy volunteers; LF, human liver fibrosis. $\mathbf{b}$ The apoptotic index from TUNEL staining was presented. $n=6$ in each group, values are presented as mean \pm SEM. ${ }^{*} P<0.05$. $\mathbf{c}$ Western blotting represented that the levels of Fas, FasL, a-SMA, COL-I, COL-IV, and cleaved caspase- 3 were enhanced in liver fibrotic sections. $\beta$-actin was used as the loading control. $\mathbf{d}$ The ratio of densitometry units of the normalized FasL/ $\beta$-actin, Fas/ $\beta$-actin, and cleaved caspase-3/ $\beta$-actin was also presented ( $n=6$ per group), values are presented as mean $\pm S E M$. ${ }^{*} P<$ 0.05 . 
$\mathbf{a}$

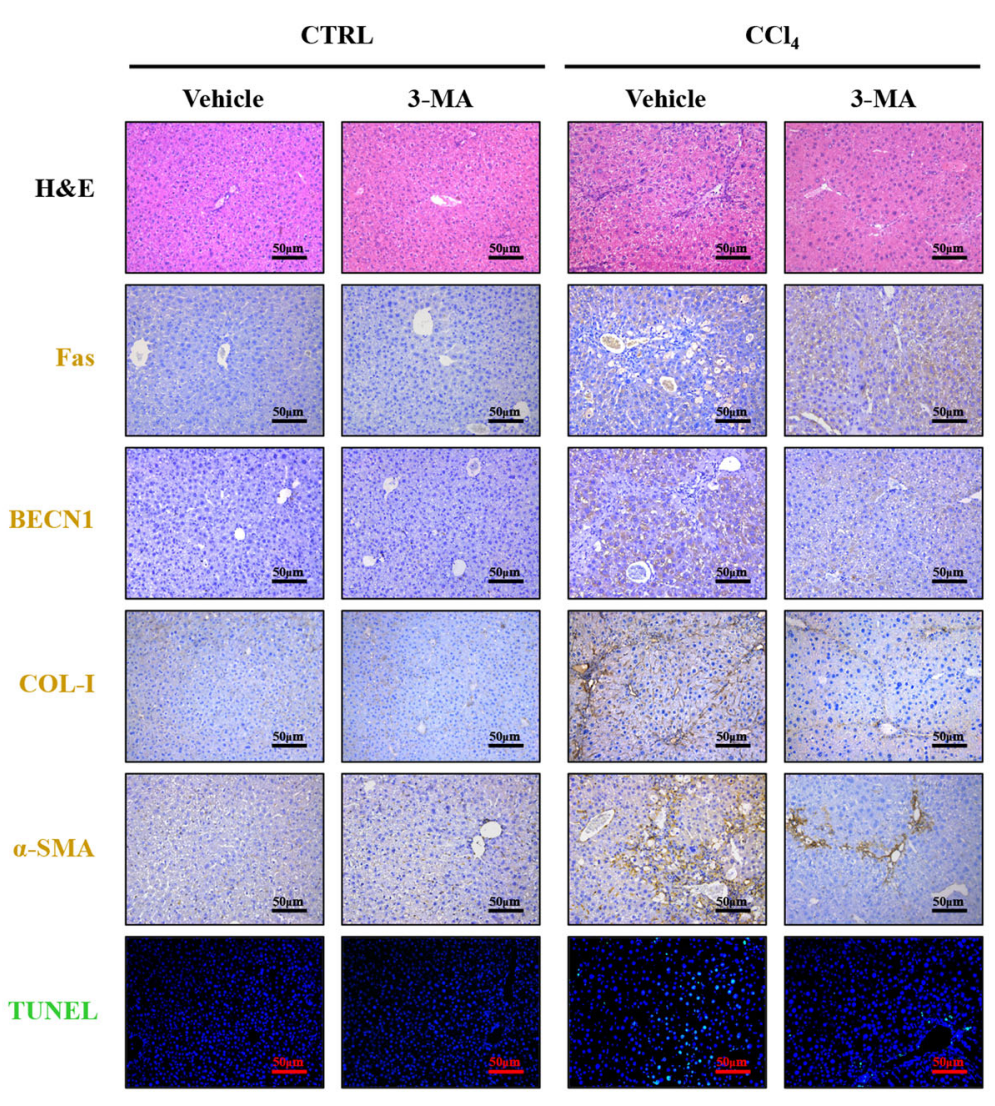

b

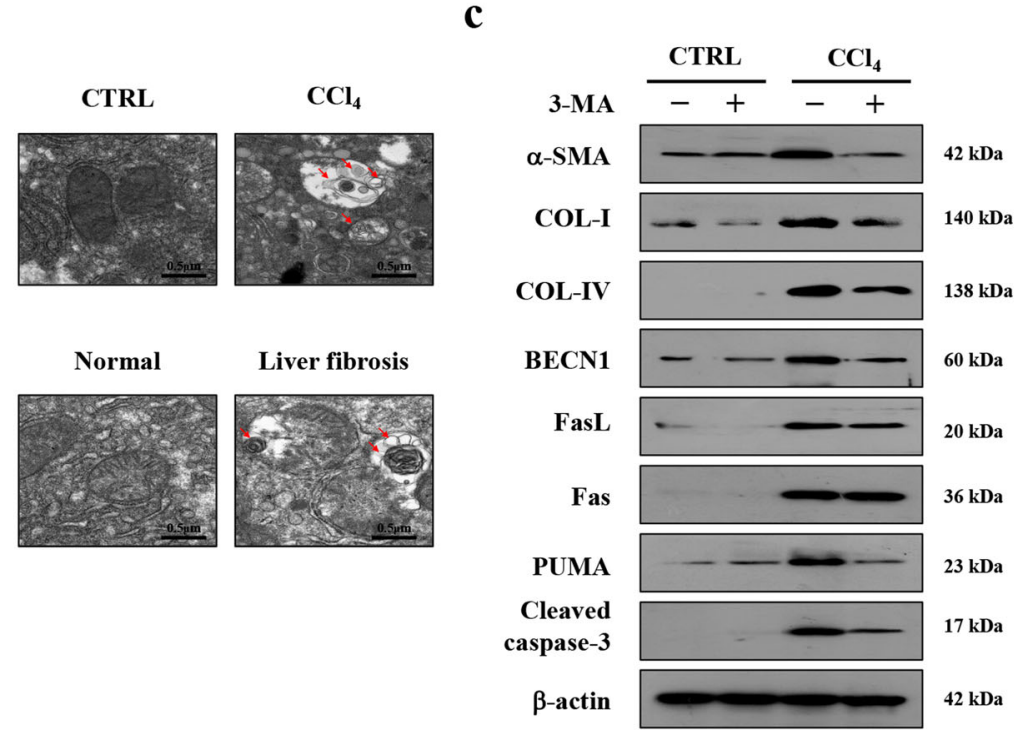

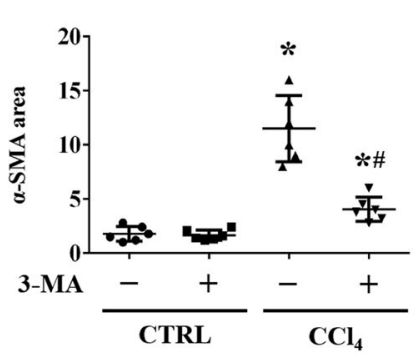
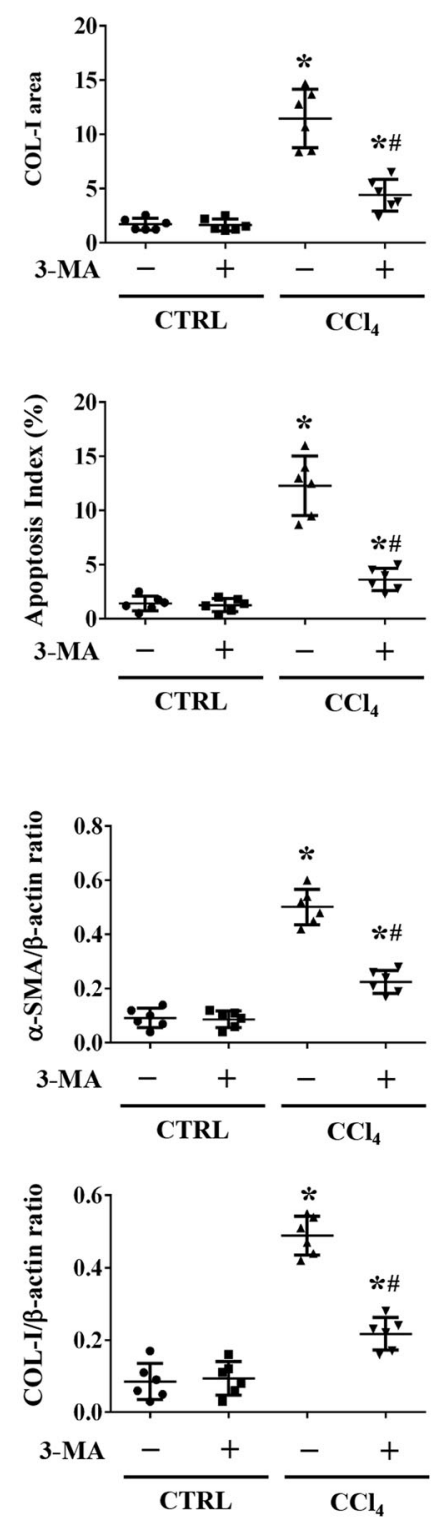

Fig. 2 Autophagy participated in Fas/FasL-mediated hepatic apoptosis in liver fibrosis. a H\&E staining, Fas, BECN1, COL-I, a-SMA staining (brown), and TUNEL staining (green) were presented in the indicated sections from olive oil- (as CTRL) or CCl - -treated mice, with or without 3-MA administration. Q-SMA area and COL-I area from the histological staining, and the apoptotic index (TUNEL staining) were also determined. ${ }^{*} P<0.05$ versus CTRL mice, ${ }^{\#} P<0.05$ versus $C_{C l}$-treated mice without 3-MA treatment, $n=6$ per group. $\mathbf{b}$ Ultrastructural features in the hepatocytes of liver tissues from human tissues and mouse models were presented (red arrows indicating autophagosomes). c Western blotting depicted that inhibition of autophagy by 3-MA downregulated the expressions of BECN1, a-SMA, COL-I, COL-IV, PUMA, and cleaved caspase-3, without affecting the state of Fas and FasL, in $\mathrm{CCl}_{4}$-treated mice. The ratio of densitometry units of the normalized a-SMA/ $\beta$-actin and COL-I/ $\beta$-actin was presented $(n=6$ per group), values are presented as mean \pm SEM. ${ }^{*} P<0.05$ versus $C T R L$ mice, ${ }^{\#} P<0.05$ versus $\mathrm{CCl}_{4}$-treated mice without 3-MA treatment. 
a

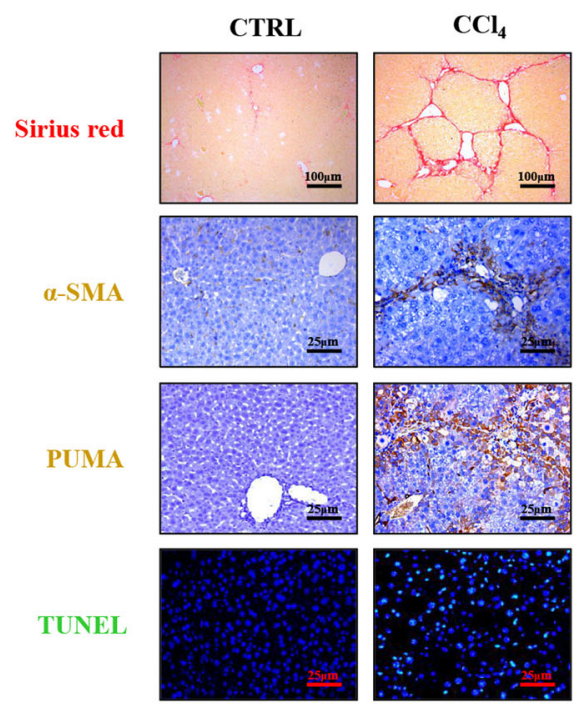

c

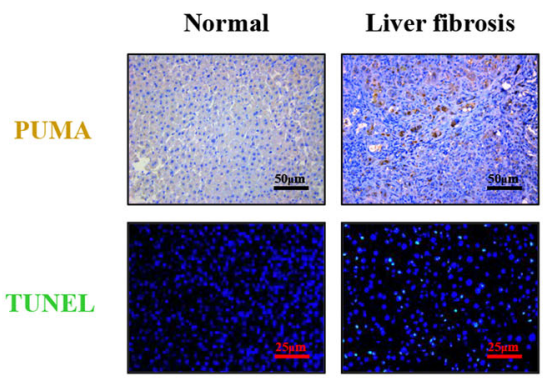

b

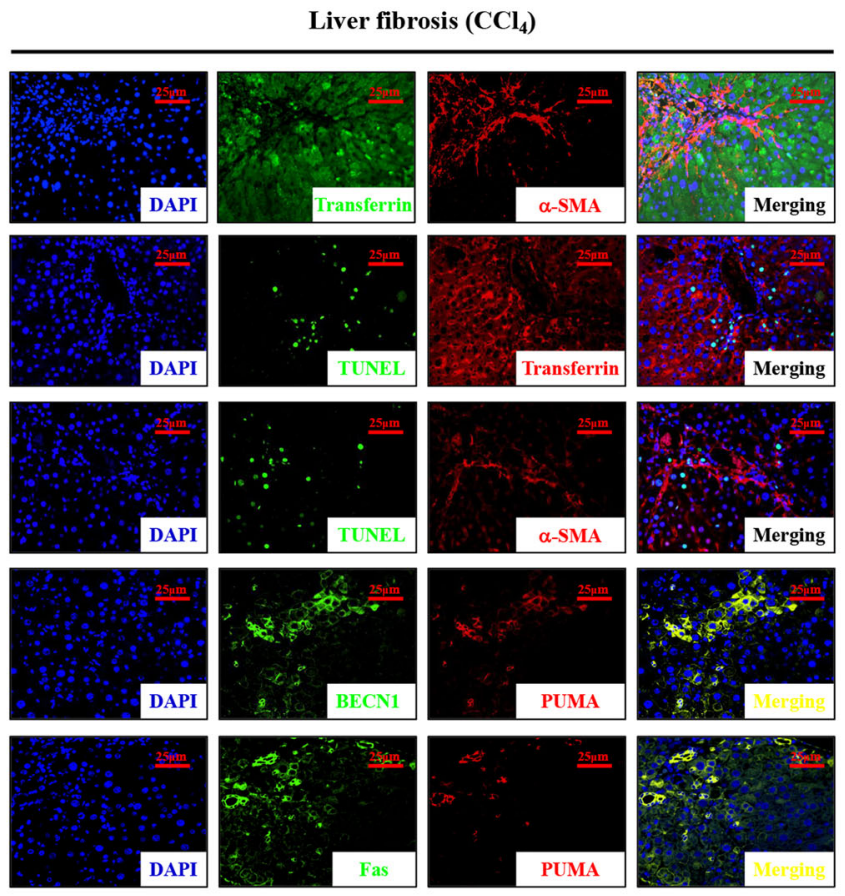

d

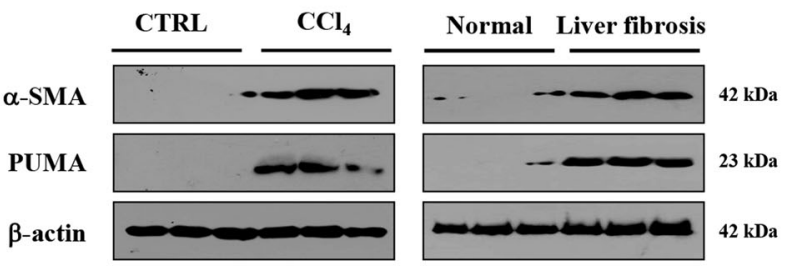

Fig. 3 PUMA responded to Fas/FasL/autophagy-mediated hepatocytes apoptosis during liver fibrosis. a Sirius red staining (red), a-SMA

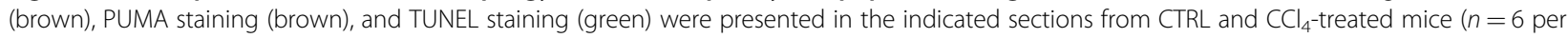
group). b Double IF staining by utilizing HSCs marker a-SMA (red) and hepatocytes marker transferrin (green) were presented (upper panel). Double staining of transferrin (red) and TUNEL (green), a-SMA (red), and TUNEL (green) indicated that the apoptotic cells mainly located in hepatocytes in liver fibrotic mice (middle panel). Co-staining of BECN1 (green) and PUMA (red), Fas (green), and PUMA (red) further revealed that PUMA involved in Fas and autophagy-regulated signaling (lower panel). Nuclei (blue) were counterstained with DAPI (4'6-diamidino-2-phenylindole dihydrochloride). c PUMA immunohistochemistry staining (brown) and TUNEL staining (green) in the indicated humans liver sections were presented. $\mathbf{d}$ PUMA and aSMA levels were determined by western blotting.

architecture and excess production and deposition of ECM, the expressions of $\alpha$-SMA, PUMA and the number of apoptotic cells (TUNEL staining) were increased in the liver fibrotic tissues (Fig. 3a). The enhanced PUMA expression and hepatic apoptosis were also observed in the human fibrotic tissues (Fig. 3c). Western blotting further revealed that the levels of $\alpha$-SMA and PUMA were obviously enhanced in the mouse and human liver fibrotic tissues (Fig. 3d). Double staining by utilizing HSCs marker $\alpha$-SMA and hepatocytes marker transferrin revealed the apoptotic cells were mainly localized in hepatocytes but not in HSCs (Fig. 3b). Co-staining of BECN1 and PUMA, Fas, and PUMA demonstrated they were mainly localized in similar cells (Fig. 3b). Western blotting showed that inhibition of autophagy by 3-MA repressed the upregulation of PUMA and caspase- 3 cleavage in $\mathrm{CCl}_{4}$-induced mouse fibrosis (Fig. 2c and Supplementary Fig. 1a).

$\mathrm{CCl}_{4}$-induced PUMA-WT and PUMA-KO liver fibrotic mouse models were adopted, and we found that deletion of PUMA ameliorated the collagen deposition, the levels 
of COL-IV, COL-I, $\alpha$-SMA, and caspase- 3 cleavage in $\mathrm{CCl}_{4}$-treated mice, although there was no obvious distinction between PUMA-WT and PUMA-KO mice from the control group (Fig. 4a). By the way, PUMA was reported to be expressed lowly or barely in normal cells and tissues, but is rapidly induced in response to a wide range of stresses in human and mouse cells ${ }^{29,31}$. Due to a very low level of PUMA in normal tissues, the low concentration of total detected proteins, and the limited detection capacity, we could not detect obvious PUMA signaling in the normal PUMA-WT mouse and further make a definite distinction between normal PUMA-WT mouse and normal PUMA-KO mouse, however, the levels of PUMA were obviously enhanced in the mouse and human liver fibrotic sections (Fig. 3a, c, d and Fig. 4a). PUMA deficiency did not affect the levels of Fas, FasL, and $\mathrm{BECN1}$, but downregulated cleaved caspase- 3 in $\mathrm{CCl}_{4^{-}}$ induced mouse fibrosis (Fig. 4b). By utilizing co-staining analysis, we found the apoptotic cells were mainly localized in hepatocytes, and deletion of PUMA decreased the number of hepatocytes apoptosis (Fig. 4c). Lastly, the activation of primary $\mathrm{HSCs}$ dissociated from $\mathrm{CCl}_{4}$-treated PUMA-WT mice was enhanced compared with that from $\mathrm{CCl}_{4}$-treated PUMA-KO mice, while PUMA deletion could not interfere with the apoptosis of primary HSCs (Fig. 4d, e). These observations revealed that PUMA contributed to Fas/FasL/autophagy-regulated hepatocytes apoptosis and HSCs activation in liver fibrosis.

\section{Fas/FasL repressed the activation of NF-KBp65 in hepatocytes in liver fibrogenesis}

Decreased or absent NF- $\mathrm{BB}$ activity in hepatocytes might lead to subsequent fibrosis by regulating hepatocytes injury and the primary trigger of fibrogenic responses in the liver, and NF-kBp65 involved in the regulation of various pathological events in chronic liver diseases $^{9}$. To investigate the correlation between Fas/ FasL and NF- $\mathrm{kBp} 65$ in liver fibrosis, liver specimens from humans and $\mathrm{CCl}_{4}$-induced mouse model were analyzed, and histological staining presented that, in contrast to the upregulation of Fas/FasL signaling in the fibrotic tissues, the activation of NF-kBp65 (phosphorylation of NF-kBp65, p-p65) was obviously repressed in the liver fibrotic tissues compared with their normal samples (Fig. 5a). Western blotting also confirmed a similar condition (Fig. 5b, c). By using primary hepatocytes isolated from the mouse models, upregulated Fas expression and downregulated p-p65 level were observed in the primary hepatocytes dissociated from $\mathrm{CCl}_{4}$-treated mice, while knockdown of Fas by siRNA significantly enhanced the activation of NF-kBp65 (p-p65) in the primary hepatocytes dissociated from $\mathrm{CCl}_{4}$-treated mice (Fig. 5d). What is more, FasL administration promoted the expression of Fas and repressed the level of p-p65 in the primary isolated hepatocytes cells, and knockdown of Fas promoted the activation of NF-kBp65 (Fig. 5e). By the way, we also found that knockdown of Fas did not obviously influence the status of NF-kBp65 and its phosphorylation in the control group, which might suggest that a very low level of Fas/FasL signaling under normal condition could not regulate NF- $\mathrm{kBp} 65$ pathway in hepatocytes. In summary, these findings indicated that Fas/FasL repressed the activation of NF-kBp65 in hepatocytes in liver fibrogenesis.

\section{NF-KBp65 inhibited PUMA-mediated hepatocytes apoptosis via $\mathrm{Bcl}-2$ family and attenuated liver fibrosis}

By using hepatocytes specific NF- $B B p 65$ deletion (p65 2 hepa) and NF- $\kappa B p 65$ wild-type $\left(p 65^{f / f}\right)$ fibrotic mouse models, TUNEL analysis performed at the different time-points of fibrosis showed that cells apoptosis was exacerbated in $\mathrm{CCl}_{4}$-treated $p 65 \Delta$ hepa mice, with an apoptotic index that was higher compared with $p 65^{f / f}$ mice. What is more, sirius red staining detected at the distinct points of fibrosis development disclosed that significant enhanced accumulation of collagen was found in $\mathrm{CCl}_{4}$-treated $p 65 \Delta$ hepa mice than $\mathrm{CCl}_{4}$-treated $p 65^{f / f}$ mice (Fig. 6a). Moreover, the phosphorylation of NFkBp65 (p-p65) was repressed in $\mathrm{CCl}_{4}$-treated $p 65^{f / f}$ mice, and p-p65-positive signaling in $p 65 \Delta$ hepa mice was only localized in the non-parenchymal cells due to hepatocytes specific $N F-\kappa B p 65$ deletion in $p 65 \Delta$ hepa mice (Supplementary Fig. 2a), these data suggest that downregulated or absent NF-kBp65 activity could lead to increase hepatocytes apoptosis in the initiation of liver fibrosis. The levels of PUMA, associates with cell apoptosis, induced by $\mathrm{CCl}_{4}$ were upregulated in the liver tissues of $p 65 \Delta$ hepa mice compared to $p 65^{f / f}$ mice (Fig. $6 \mathrm{~b}, \mathrm{c}$ ). Double staining of TUNEL and PUMA, TUNEL, and transferrin indicated that PUMA and TUNEL signaling were mainly located in hepatocytes of $\mathrm{CCl}_{4}$-treated $p 65 \Delta$ hepa mice (Fig. 6b). Furthermore, we found that the activation (detected by $\alpha$ SMA) of primary HSCs rather than their death (detected by cleaved caspase-3) was visibly enhanced in $\mathrm{CCl}_{4}$-treated $p 65 \Delta$ hepa mice than that in $p 65^{f / f}$ mice (Fig. $6 \mathrm{~d}, \mathrm{e}$ ).

The pro-survival $\mathrm{Bcl}-2$ family members actively sequester the pro-apoptotic BH3-only members, like PUMA, and finally inhibit the induction of apoptosis ${ }^{36-38}$. By microanalysis screening for primary hepatocytes from mouse models, we found that PUMA (BBC3) in primary hepatocytes from fibrotic tissues presented a higher expression than that from normal tissues, while the $\mathrm{Bcl}-2$ family members were not affected, it seems that the unchanged Bcl-2 family could not be enough to suppress the detrimental effects of PUMA during liver fibrosis (Supplementary Fig. 2b). Western blotting revealed that the expressions of $\mathrm{Bcl}-2, \mathrm{Bcl}-\mathrm{xL}$, and $\mathrm{Mcl}-1$ were downregulated in the primary hepatocytes of $p 65 \Delta$ hepa fibrotic 


\section{$\mathbf{a}$}

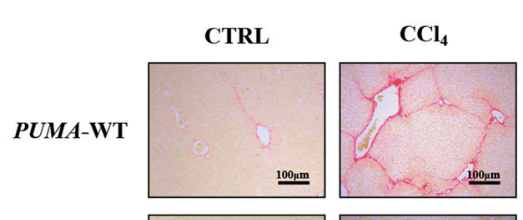

PUMA-KO

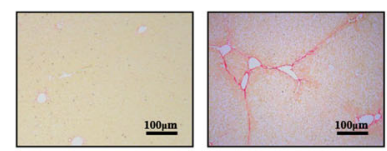

Sirius red

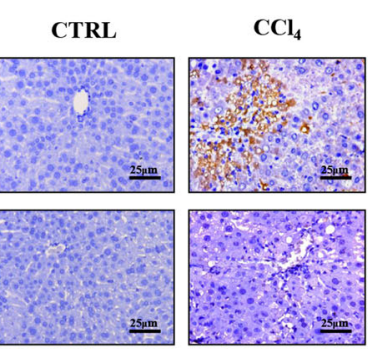

Cleaved caspase- 3

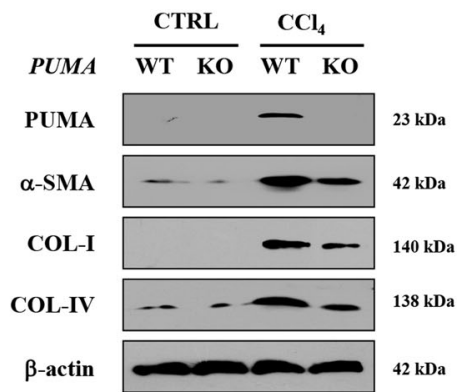

b
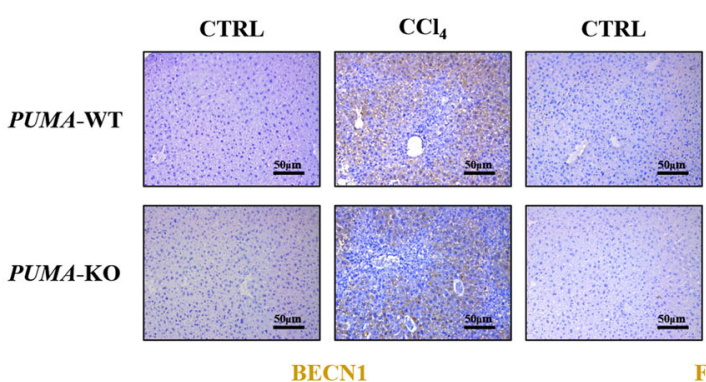

$\mathrm{CCl}_{4}$
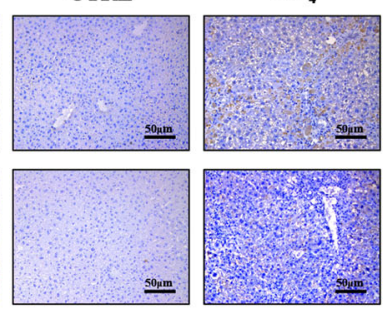

Fas

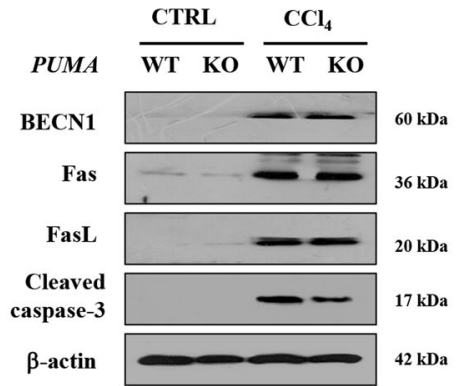

c

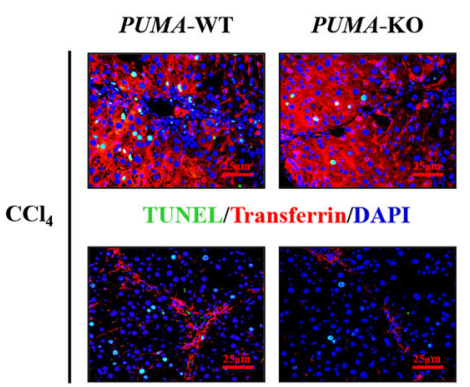

TUNEL/a-SMA/DAPI

d
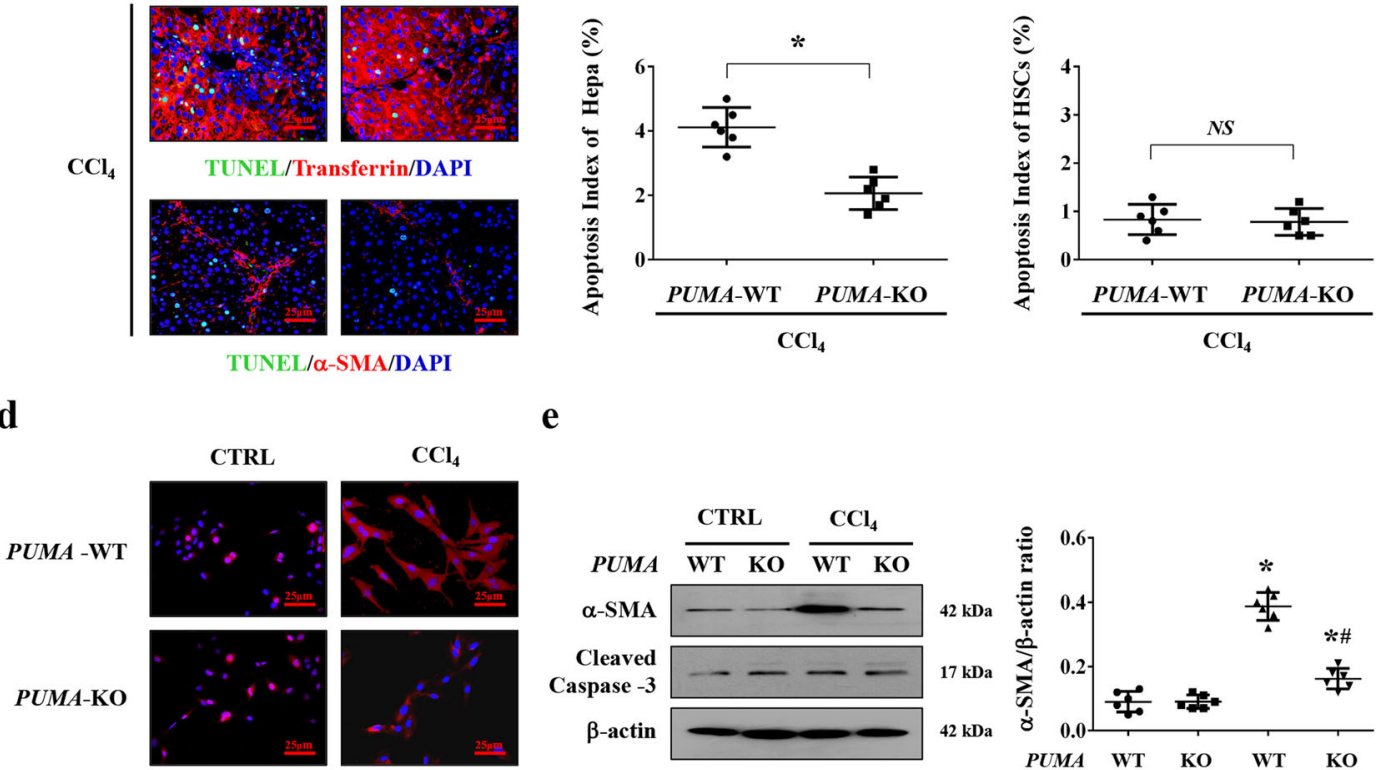

$\alpha-$ SMA/DAPI

e

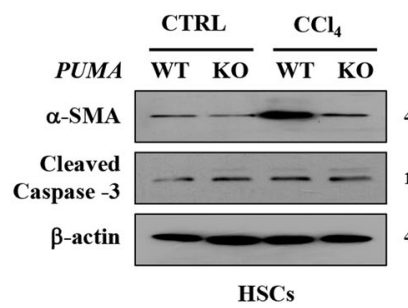

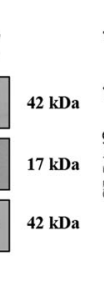

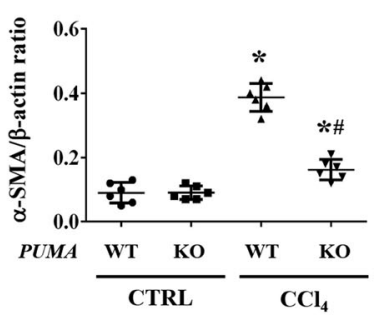

Fig. 4 Targeted deletion of PUMA ameliorated hepatocytes apoptosis and liver fibrosis. a Sirius red staining (red) and cleaved caspase-3 staining (brown) presented that targeted deletion of PUMA ameliorated hepatic apoptosis and collagen deposition. Western blotting presented that

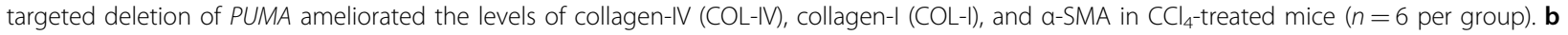
Immunohistochemistry staining and western blotting revealed that PUMA deficiency did not affect the status of Fas and BECN1 in CCl -treated mice. c Double immunofluorescence staining (transferrin (red) and TUNEL (green), a-SMA (red) and TUNEL (green)) and the analysis of the apoptotic index of hepatocytes or HSCs indicated that targeted deletion of PUMA mainly ameliorated hepatocytes apoptosis during liver fibrosis, $n=6$ per group. Nuclei (blue) were counterstained with DAPI. ${ }^{*} P<0.05$. NS, no significance. $\mathbf{d}$ a-SMA (red) staining in the primary HSCs dissociated from the indicated PUMA-WT and PUMA-KO mice was represented, nuclei (blue) were counterstained with DAPI. e The indicated proteins from primary HSCs analyzed by western blotting. The ratio of densitometry units of the normalized a-SMA/ $\beta$-actin was also determined, $n=6$ per group, values are presented as mean \pm SEM. ${ }^{*} P<0.05$ versus primary HSCs from CTRL mice, ${ }^{\#} P<0.05$ versus primary HSCs from $C_{C C l}$-treated $P U M A-W T$ mice. 
$\mathbf{a}$

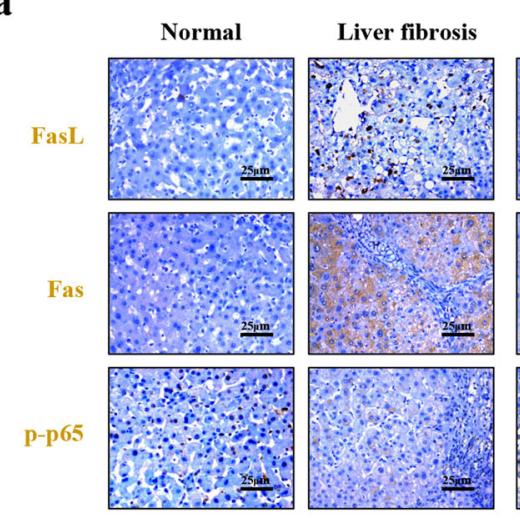

c

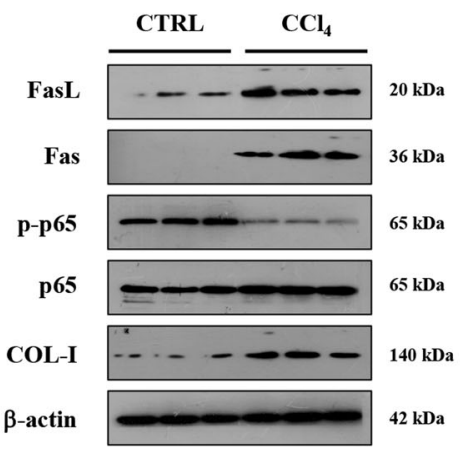

d

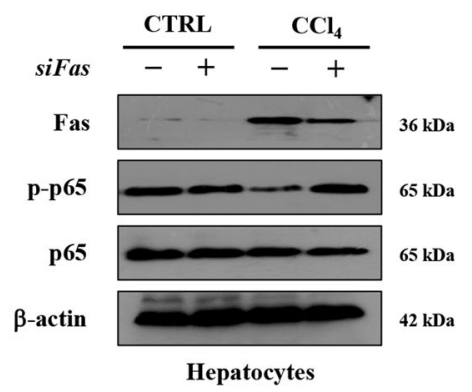

e

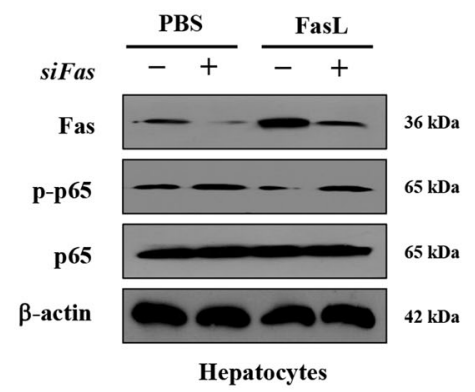

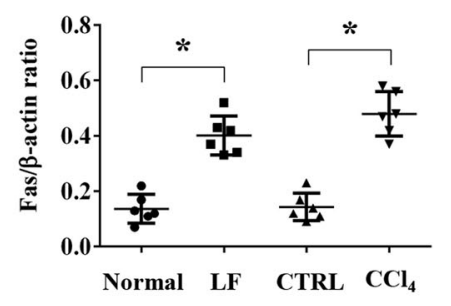
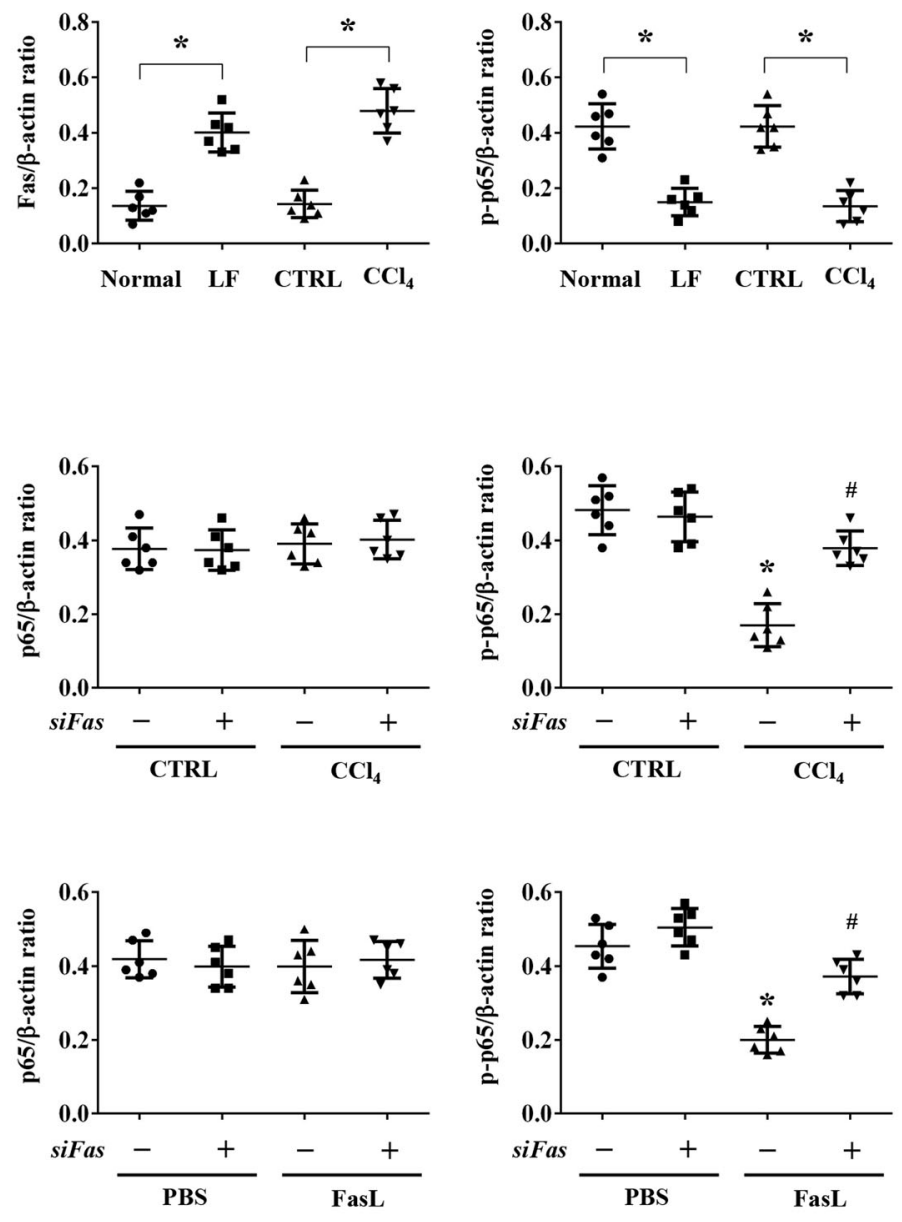

Fig. 5 (See legend on next page.) 
(see figure on previous page)

Fig. 5 Fas/FasL repressed the activation of NF-KBp65 in hepatocytes in liver fibrogenesis. a Immunohistochemical staining (brown) of Fas, FasL, and p-p65 (the phosphorylation of NF-KBp65) in the indicated liver sections was presented ( $n=6$ per group). b Expressions of Fas, FasL, p65, pp65, and COL-I proteins from the related humans liver sections were detected by western blotting. c Western blotting was adopted to analyze the levels of Fas, FasL, p65, p-p65, and COL-I in the liver tissues of mouse models. The ratio of densitometry units of the normalized Fas/ $\beta$-actin and pp65/ $\beta$-actin from (b) and (c) was also determined, $n=6$ per group, values are presented as mean $\pm S E M$. ${ }^{*} P<0.05$. CTRL, the control mice (olive oiltreated mice); $\mathrm{CCl}_{4}, 20 \%$ carbon tetrachloride-induced mouse fibrosis; Normal, healthy volunteers; LF, human liver fibrosis. $\mathbf{d}$ Western blotting depicted that knockdown of Fas upregulated the level of p-p65 in the primary hepatocytes isolated from $\mathrm{CCl}_{4}$-treated mice. The ratio of densitometry units of the normalized $p 65 / \beta$-actin and $p$-p65/ $\beta$-actin was also presented, $n=6$ per group, values are presented as mean \pm SEM. ${ }^{*} P<0.05$ versus primary hepatocytes from CTRL group, ${ }^{\#} P<0.05$ versus primary hepatocytes from $\mathrm{CCl}_{4}$-treated group without siFas treatment. e FasL treatment enhanced the expression of Fas and repressed the phosphorylation of NF-KBp65 ( $\mathrm{p}-\mathrm{p} 65$ ) in the primary hepatocytes, while knockdown of Fas by siRNA upregulated the level of p-p65 in FasL-treated group. The ratio of densitometry units of the normalized p65/ $\beta$-actin and p-p65/ $\beta$-actin was also presented, $n=6$ per group, values are presented as mean \pm SEM. ${ }^{*} P<0.05$ versus primary hepatocytes from PBS group, ${ }^{\#} P<0.05$ versus primary hepatocytes from FasL-treated group without siFas treatment.

mice (Fig. 6c). Adopting the Bcl-2 inhibitor ABT-199, we revealed that the expressions of PUMA and $\alpha$-SMA, along with enhanced hepatocytes apoptosis and collagen deposition, were upregulated compared to that without ABT-199 administration (Supplementary Fig. 2c). By the way, the phosphorylation of NF-kBp65 (p-p65) was repressed in primary hepatocytes isolated from $\mathrm{CCl}_{4}$ treated $p 65^{f f f}$ mice compared to those from the control group (Fig. 6c), while ABT-199 did not influence the phosphorylation of NF- $\mathrm{kBp} 65$ in primary hepatocytes both from the control group and $\mathrm{CCl}_{4}$-treated $p 65^{f / f}$ mice (Supplementary Fig. 2d). Lastly, primary HSCs analysis suggested that ABT-199 administration promoted HSCs activation in liver fibrosis (Supplementary Fig. 2e), These data suggested that NF-kBp65 regulated PUMA-mediated hepatocytes apoptosis and liver fibrosis via Bcl-2 family.

\section{NF-kBp65/PUMA-regulated hepatocytes apoptosis drove inflammatory response to promote HSCs activation and liver fibrosis}

Dying hepatocytes could release alarmins and activate inflammatory cells to produce various inflammatory cytokines to contribute to the initiation and development of liver fibrosis ${ }^{9,10}$. Based on these, after $\mathrm{CCl}_{4}$ treatment, the hepatic inflammatory cells or mediators, including myeloperoxidase (MPO, neutrophil), F4/80 (macrophages), TNF- $\alpha$, TGF- $\beta$, IL- 6 , and CD3 (lymphocytes) were upregulated in PUMA-WT mice compared to PUMA-KO mice (Fig. 7a). The inflammatory cytokines mRNA, including $I L-1 \alpha, I L-6, T N F-\alpha, I L-1 \beta, T G F-\beta$, and $I L-10$, were also enhanced in $\mathrm{CCl}_{4}$-treated PUMA-WT mice (Fig. 7b). Furthermore, MPO, F4/80, TNF- $\alpha$, TGF- $\beta$, IL-6, and CD3 were upregulated in $p 65 \Delta$ hepa mice compared to $p 65^{f / f}$ mice, while PUMA antisense oligonucleotides (AS) treatment suppressed this inflammatory response (Figs. 7c and $8 \mathrm{a}$ ). By adopting the TGF- $\beta 1$ inhibitor pirfenidone, TNF- $\alpha$ inhibitor pentoxifylline, and monocytes depressor clodronate-loaded liposomes in p65 $\mathrm{h}$ hepa/PUMA-WT mice, we found inhibition of inflammatory action ameliorated liver fibrosis (Fig. 8b, c). $\alpha$-SMA levels in primary $\mathrm{HSCs}$ isolated from the $\mathrm{CCl}_{4}$ treated $p 65 \Delta$ hepa/PUMA-WT mice were visibly upregulated, while above inhibitors administration could hold back the activation of HSCs (Fig. 8b, d). These data indicated that PUMA-promoted HSCs activation depended on the inflammatory response following hepatocytes apoptosis.

Following the induction of liver fibrosis in $\mathrm{CCl}_{4}$-induced PUMA-WT and PUMA-KO mouse models, these mice serum was extracted respectively (Fig. 9a). The inflammatory mediators mRNA levels from PUMA-WT mice, including $I L-1 \alpha, I L-6, T N F-\alpha, I L-1 \beta, T G F-\beta$, and $I L-10$, were higher than that from PUMA-KO mice (Fig. 9b). The primary HSCs (isolated from normal PUMA-WT mice) accepted the serum of PUMA-WT liver fibrotic mice showed enhanced expressions of desmin, vimentin, $\alpha$ SMA, and PCNA (proliferating cell nuclear antigen), in contrast to the HSCs accepting PUMA-KO mice serum (Fig. 9c). Similar changes in the HSCs activation and growth elements were also observed by western blotting analysis (Fig. 9d). By in vivo serum test, the serum was extracted from $\mathrm{CCl}_{4}$-induced liver fibrosis models in PUMA-WT and PUMA-KO mice, respectively, and then was transfused to the different PUMA-KO littermates via tail intravenous injection (Fig. 9e). Following that, mice who accepted PUMA-WT serum appeared increased collagen deposition and enhanced HSCs activation rather than apoptosis, compared to that accepted PUMA-KO serum (Fig. 9f, g). To sum up, these data demonstrated that NF-kBp65/PUMA-regulated hepatocytes apoptosis drove inflammatory response to promote HSCs activation and liver fibrosis.

\section{Discussion}

Hepatocytes apoptosis has been considered to be in a series of biochemical steps in most forms of liver injury under a number of pathological conditions ${ }^{10}$. Regulation of hepatocytes apoptosis is crucial for liver homeostasis 
$\mathbf{a}$
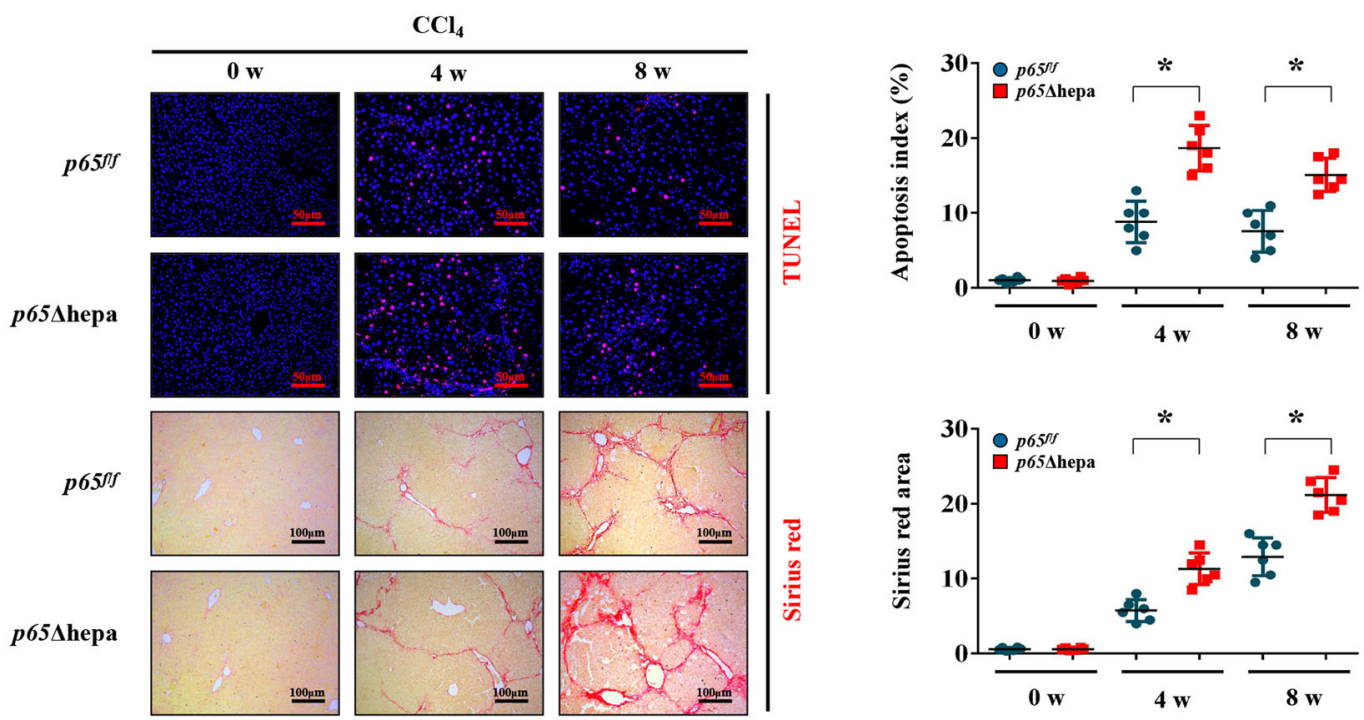

b
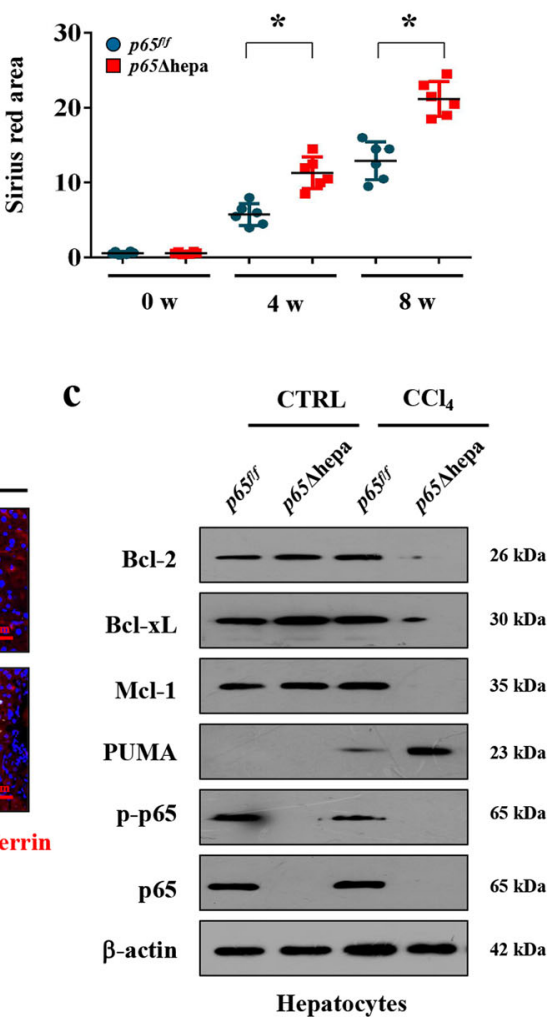

d
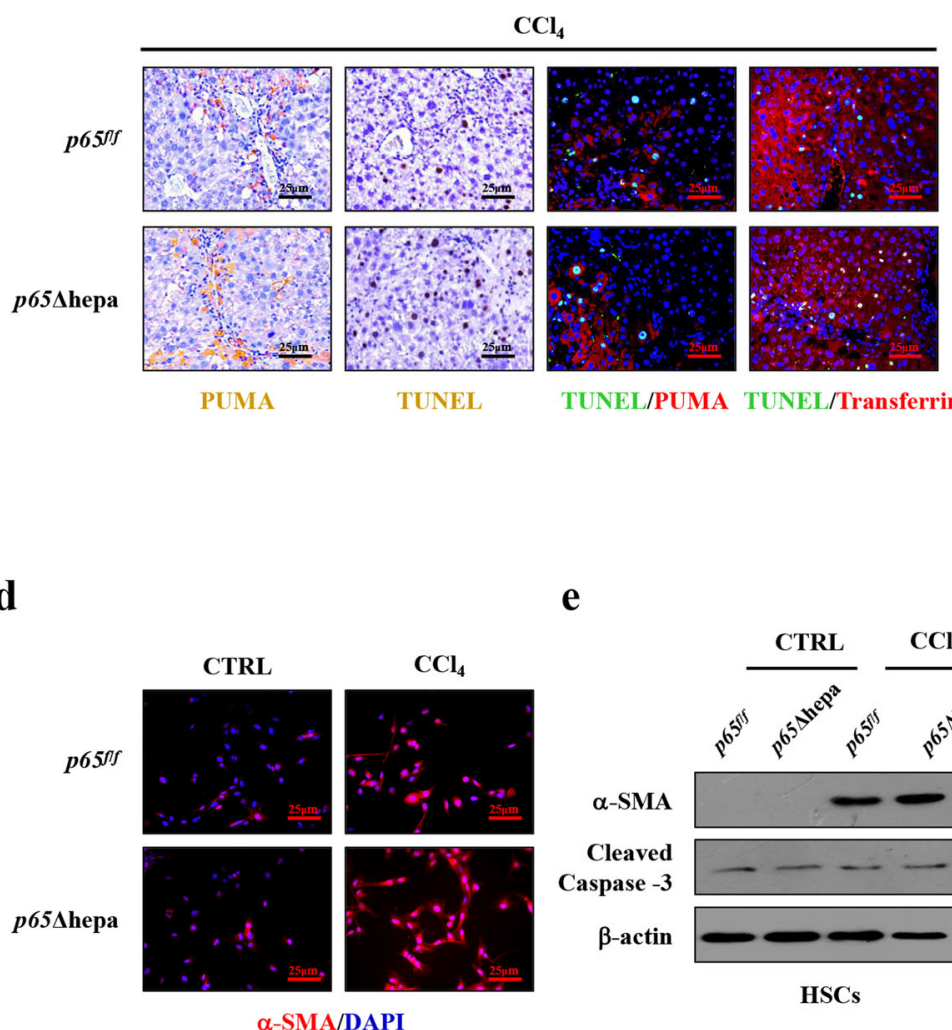

e
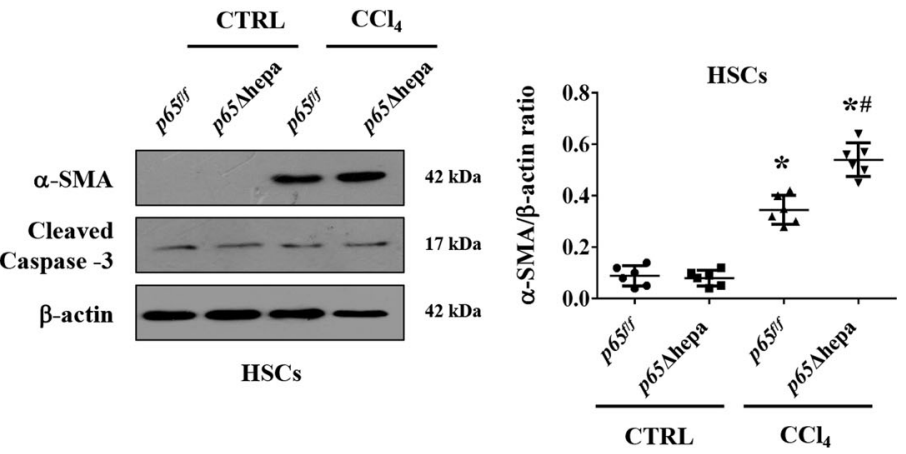

Fig. 6 (See legend on next page.) 
(see figure on previous page)

Fig. 6 NF-KBp65 inhibited PUMA-mediated hepatocytes apoptosis and attenuated liver fibrosis. a TUENL staining (red) and sirius red staining at the indicated time points were adopted to showed that NF-KBp65 deficiency in hepatocytes promoted apoptosis and collagen deposition during fibrogenesis. The apoptotic index and sirius red area were also analyzed. ${ }^{*} P<0.05$. $p 65 \Delta$ hepa: hepatocytes specific NF-KBp65 deletion; $p 65^{f / f}$ : NF-KBp65 wild-type. $\mathbf{b}$ Immunohistochemical staining revealed NF-KBp65 deficiency in hepatocytes induced PUMA expression and cell apoptosis (brown). Double staining of TUNEL (green) and PUMA (red) indicated that PUMA expression and TUNEL signaling were located in similar cells, and co-staining of TUNEL (green) and transferrin (red) was also presented. Cell nuclei (blue) were counterstained by DAPI. c Western blotting represented hepatocytes specific NF-KBp65 deletion promoted the upregulation of PUMA and downregulated the levels of anti-apoptotic proteins Bcl-2, BCl-xL, and $\mathrm{MCl}-1$ in primary hepatocytes isolated from $\mathrm{CCl}_{4}$-treated mice, and the phosphorylation of NF-kBp65 ( $\mathrm{p}$-p65) was repressed in primary hepatocytes isolated from $\mathrm{CCl}_{4}$-treated $p 65^{f / f}$ mice. $\mathbf{d}$ Primary HSCs dissociated from the indicated mice were stained by a-SMA (red). Nuclei (blue) were counterstained with DAPI. e The indicated proteins from primary isolated HSCs in the mouse models were detected by western blotting. The ratio of densitometry units of the normalized a-SMA/ $\beta$-actin was also determined. ${ }^{*} P<0.05$ versus primary HSCs from CTRL mice, ${ }^{\#} P<0.05$ versus HSCs from $\mathrm{CCl}_{4}$-treated $p 65^{\mathrm{f} / \mathrm{f}}$ mice. $n=6$ per group.

and increased sensitivity of hepatocytes towards apoptosis results in chronic liver injury, which is linked to trigger inflammatory and wound healing responses that in the long run promote the development of hepatic fibrosis ${ }^{10}$. So far, three classic pro-apoptotic networks, TNF- $\alpha /$ TNF receptor 1 (TNFR1), Fas/FasL, and TNF-related apoptosis-inducing ligand (TRAIL)/death receptor 4 (DR4 or TRAIL-R1)/death receptor 5 (DR5 or TRAIL-R2), have been widely demonstrated to mediate caspase-dependent cellular apoptosis $^{39,40}$. Our previous research has revealed that autophagic flux involved in liver fibrogenesis ${ }^{21}$. Autophagy has long been recognized as a critical pathway in the regulation of cell death, and the autophagic machinery could directly interface with pro-apoptotic factors pathways to promote cell death ${ }^{14,21}$. Several studies have focused on the role of Fas/FasL signaling during autophagy, and Fas/FasL has been verified to mediate autophagy activation in cell line systems including human neuroblastoma (SH-SY5Y) cells, human lung cancer SPCA-1 cells and HeLa cells, and Fas signaling could activate autophagic cascades in retina-retinal pigment epithelium (RPE) separation during retinal detachment via regulating the conversion of LC3-I to LC3-II and the expression of Atg5. Furthermore, inhibition of autophagy by 3-MA could obviously reverse the outcome mediated by Fas/FasL signaling ${ }^{41-43}$. They also revealed that Fas/FasL promoted the activation of autophagy via modulating Fas-activated death domain (FADD), Src, the c-Jun N-terminal kinase (JNK) family of stress kinases, BECN1, PI3K, etc ${ }^{41-43}$. Given the critical roles that Fas/FasL signaling and autophagy play in liver diseases, in this study, by analyzing the liver sections from both humans and mice, we uncovered that Fas/ FasL presented distinctly higher expression in the fibrotic tissues than in normal liver sections, and Fas/ FasL signaling is upstream of autophagy, while inhibition of autophagy ameliorated Fas/FasL-regulated hepatic apoptosis, $\alpha$-SMA expression, and liver fibrosis, and this data suggested that Fas/FasL-mediated hepatic apoptosis by autophagy in liver fibrogenesis.
Autophagy has been reported to control the timing of mitochondrial permeabilization in apoptosis and selectively regulate PUMA level, while PUMA depletion prevents sensitization to apoptosis by autophagy inhibition ${ }^{44,45}$. PUMA transduces death signals primarily to the mitochondrial membrane to lead to caspase activation and ultimately cell death via interaction and inhibition of the anti-apoptotic Bcl-2 repertoire ${ }^{46}$. In our study, we found that, accompanying by the loss of preserved architecture and excess deposition of ECM, PUMA was induced in liver fibrosis, and deletion of PUMA attenuated HSCs activation and liver fibrosis via inhibiting hepatocytes apoptosis, without influencing the status of the Fas/FasL signaling and autophagy. Moreover, inhibition of autophagy repressed PUMA upregulation and hepatocytes apoptosis to alleviate liver fibrosis, and we suggested that PUMA contributed to the Fas/FasL/ autophagy-regulated hepatocytes apoptosis and HSCs activation in liver fibrosis.

NF-kBp65 has a wide range of functions in different cellular compartments influencing the survival of hepatocytes and the activation of $\mathrm{HSCs}^{47}$. Recent study has suggested that miR-196b-5p-mediated downregulation of Fas involved in the activation of STAT3 signaling through the NF-kBp65/IL-6 axis, and Fas downregulation could activate NF- $\mathrm{kBp} 65$ signaling to promote lung cancer cell growth in non-small cell lung cancer (NSCLC) ${ }^{48}$, which powerfully demonstrated the interaction between Fas/ FasL and NF-kBp65. In our study, we also verified the correlation between them and found that the activation of NF-kBp65 (phosphorylation of NF-kBp65, p-p65) was repressed in the liver fibrotic tissues compared with their normal samples. By using primary hepatocytes, we further demonstrated that upregulated Fas and downregulated pp65 were observed in FasL-treated hepatocytes and in the primary hepatocytes dissociated from $\mathrm{CCl}_{4}$-treated mice, while knockdown of Fas could significantly reverse that and enhance the activation of NF- $\mathrm{kBp} 65$, indicating that Fas/FasL signaling repressed the activation of NF-kBp65 in hepatocytes in liver fibrogenesis. Furthermore, we 
a

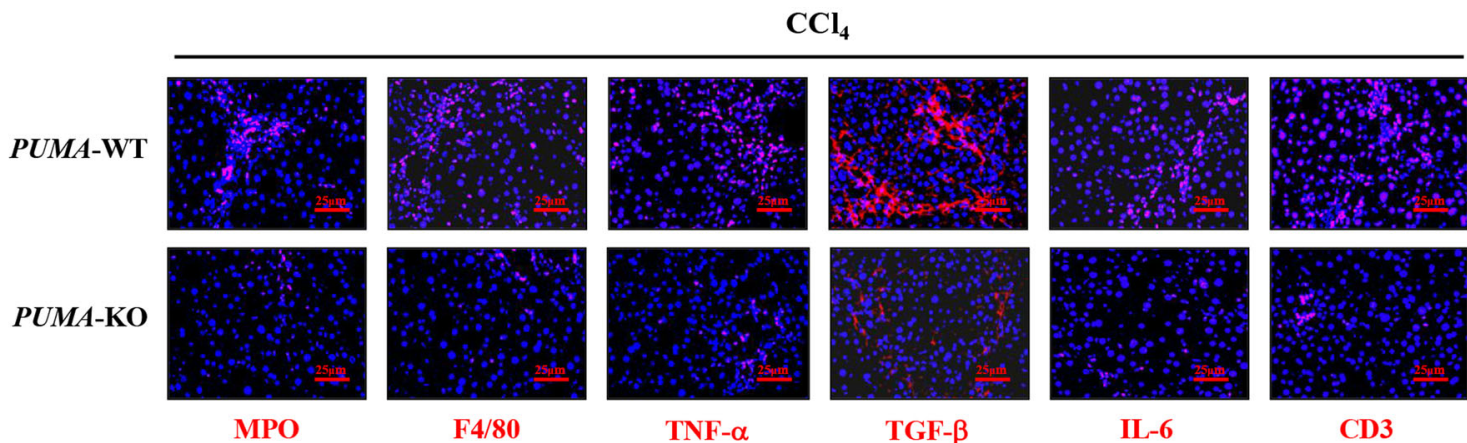

b
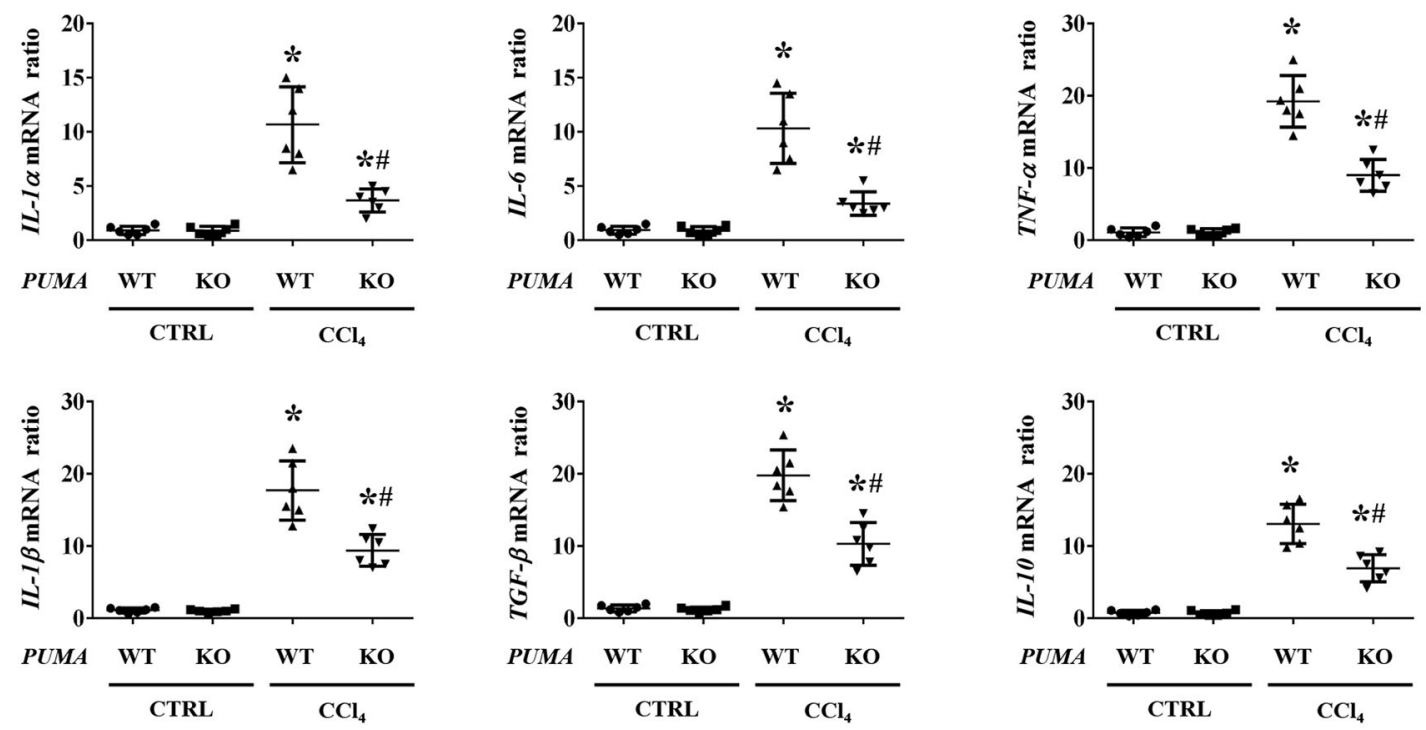

c

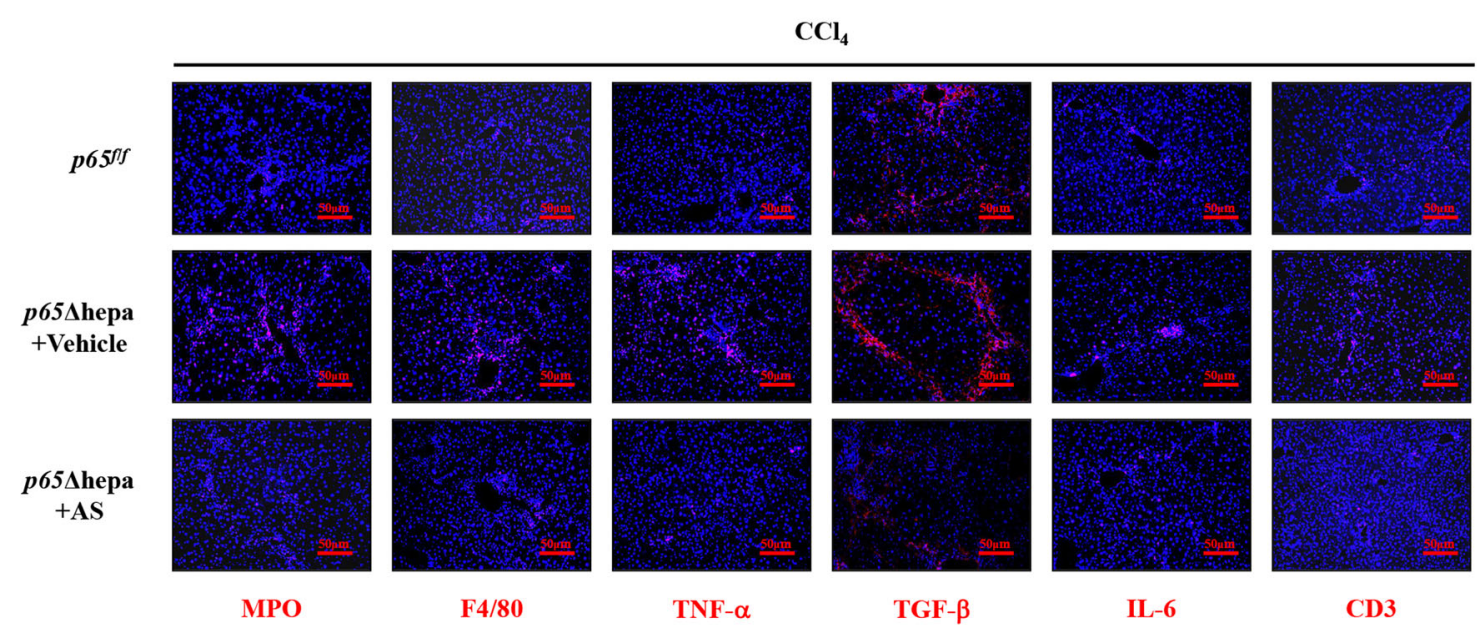

Fig. 7 NF-KBp65/PUMA-regulated hepatocytes apoptosis enhanced hepatic inflammatory response. a The indicated inflammatory cytokines in the mouse livers were analyzed via immunofluorescence staining (red), the related cytokines were significantly repressed in CCl -treated $P U M A-K O^{-}$ mice, nuclei (blue) were counterstained with DAPI. b Inflammatory factors mRNA expression in the livers from either CCl 4 -treated PUMA-WT or PUMA$\mathrm{KO}$ mice were analyzed by quantitative reverse-transcription PCR. ${ }^{*} P<0.05$ versus CTRL mice, ${ }^{\#} P<0.05$ versus $C \mathrm{Cl}_{4}$-treated $P U M A-W T$ mice. The expression of $\beta$-actin in each tissue was quantified as the internal control. $n=6$ per group. $\mathbf{c}$ Expressions of indicated inflammatory cytokines in the livers were analyzed via immunofluorescence staining (red), the related cytokines were repressed in $\mathrm{CCl}_{4}$-treated p65 $\triangle$ hepa mice following PUMA antisense oligonucleotides (AS), nuclei (blue) were counterstained with DAPI. 
a
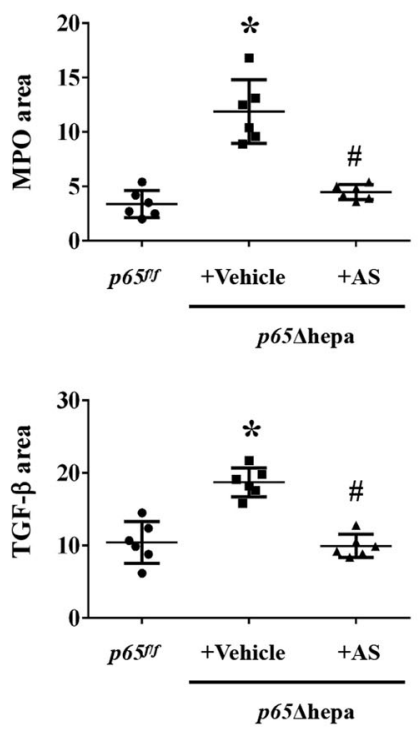
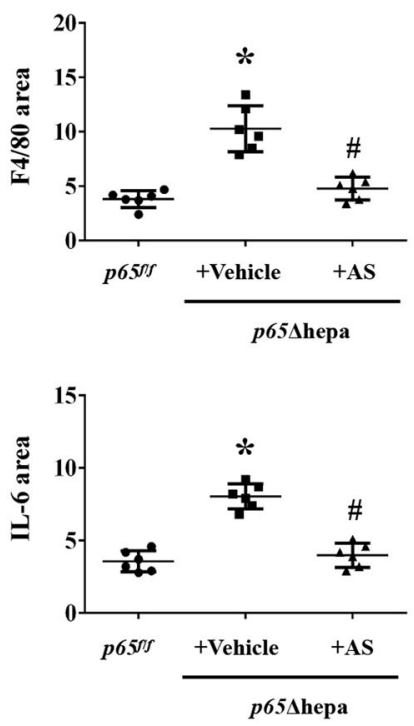
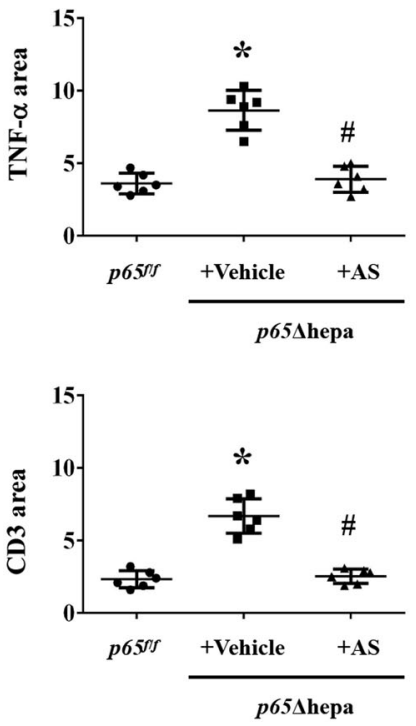

b

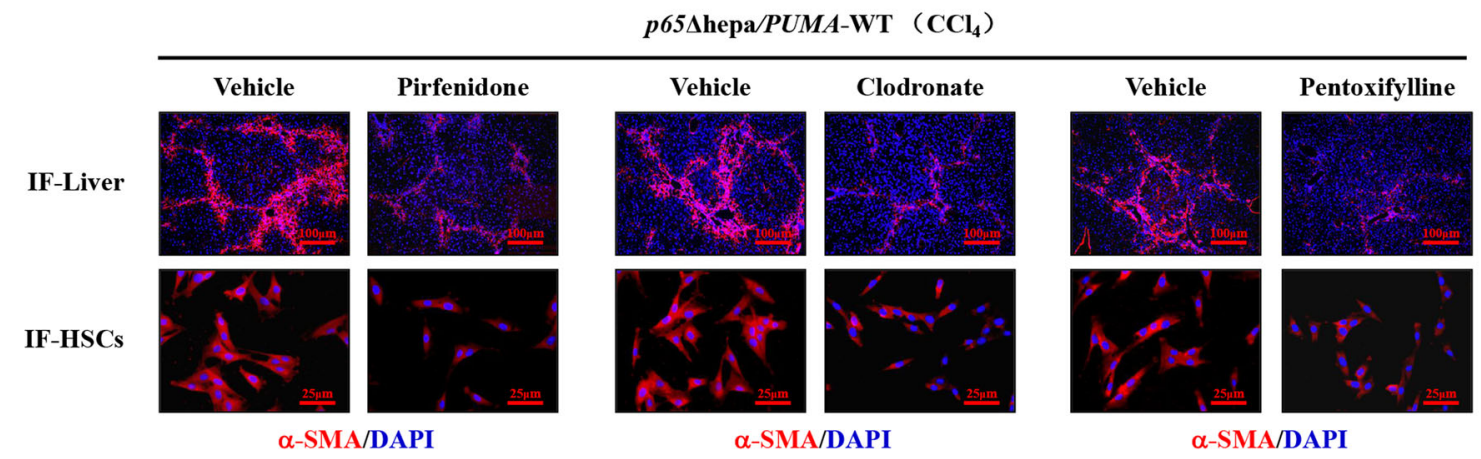

C

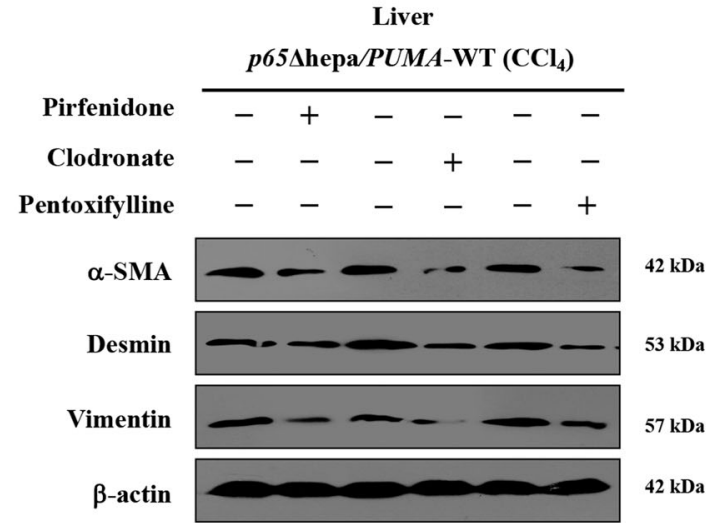

d

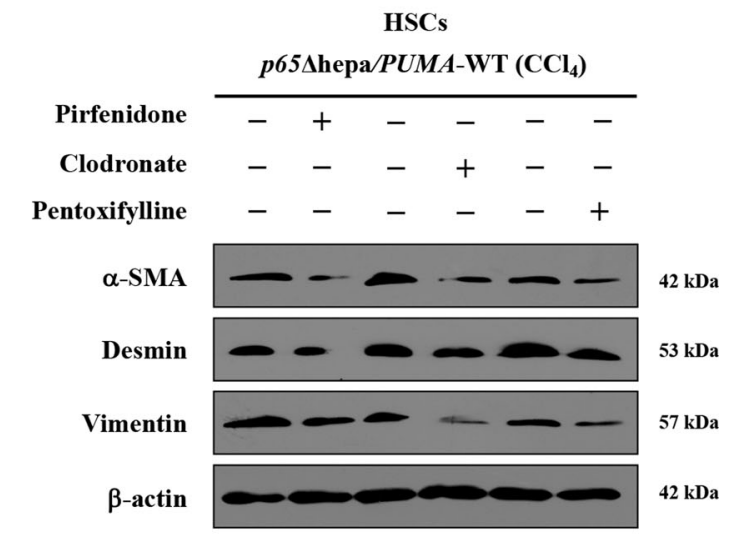

Fig. 8 NF-KBp65/PUMA-regulated hepatocytes apoptosis-linked inflammatory response to promote HSCs activation and liver fibrosis. a The area of the indicated inflammatory cytokines from immunofluorescence staining of Fig. $7 c$ was analyzed. ${ }^{*} P<0.05$ versus $p 65^{f / f}$ mice, ${ }^{\#} P<0.05$ versus p65 $\triangle$ hepa mice with vehicle administration. $\mathbf{b}$ The administration of TGF- $\beta 1$ inhibitor pirfenidone, TNF-a inhibitor pentoxifylline or monocytes inactivator clodronate-loaded liposomes, attenuated HSCs activation (a-SMA staining of primary HSCs, red) and liver fibrosis (a-SMA staining, red) in p65 $\triangle$ hepa/PUMA-WT mice. Nuclei (blue) were counterstained with DAPI, $n=6$ per group. IF, immunofluorescence staining. $\mathbf{c}$, $\mathbf{d}$ Western blotting presented that pirfenidone, pentoxifylline, or clodronate-loaded liposomes attenuated liver fibrosis (liver section) and the activation of HSCs (primary HSCs section) in $\mathrm{CCl}_{4}$-induced $p 65 \triangle \mathrm{hepa} / \mathrm{PUMA-WT}$ mouse model, respectively. $n=6$ per group. 
$\mathbf{a}$

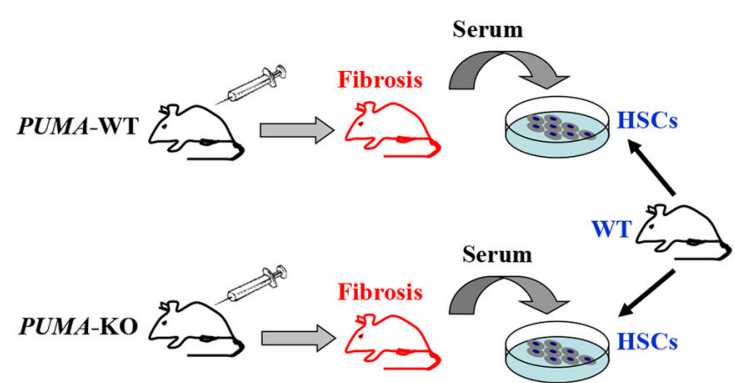

b

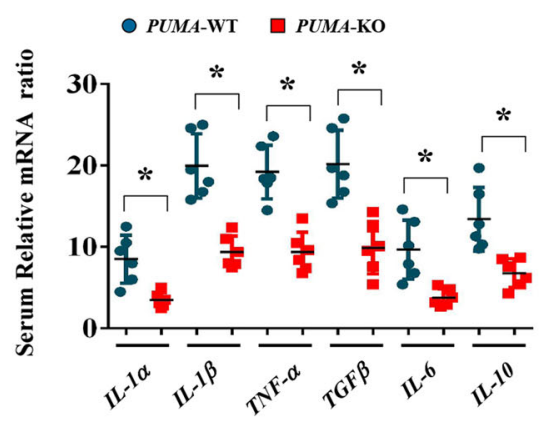

c

PUMA-WT HSCs
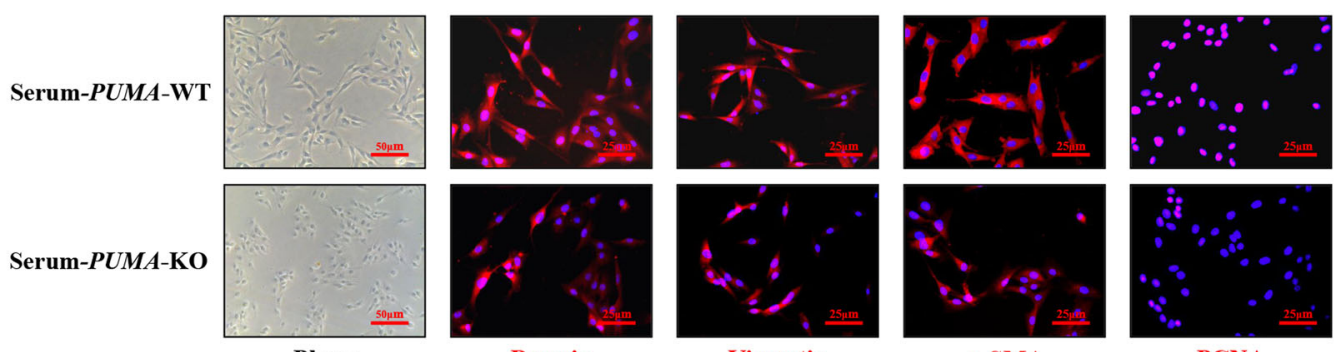

Vimentin

$\alpha$-SMA

PCNA

d

e
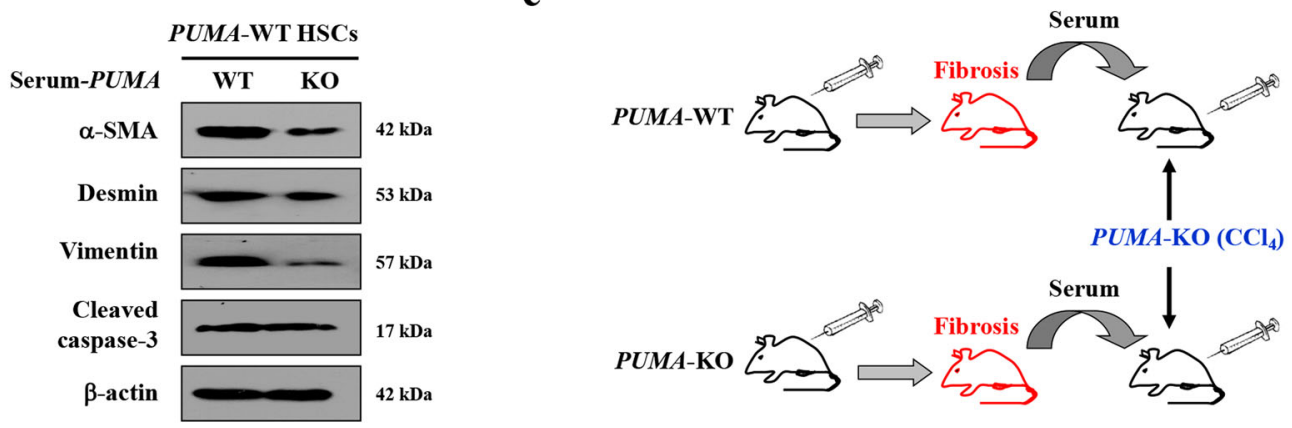

f

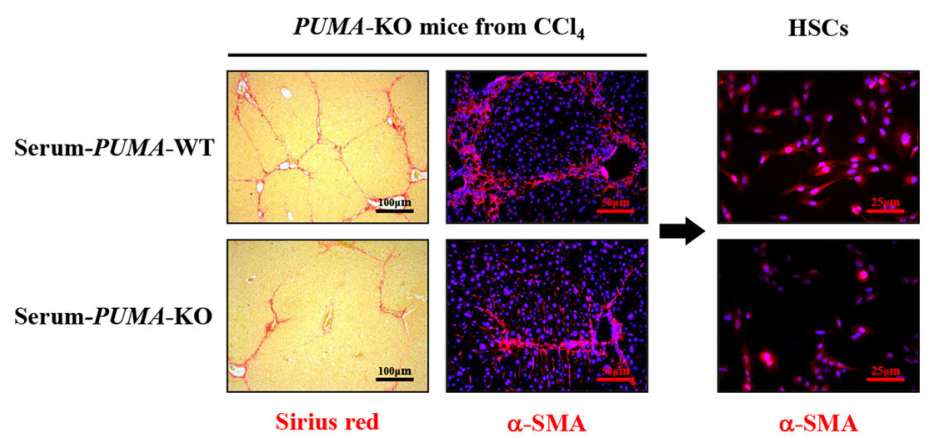

g

PUMA-KO HSCS

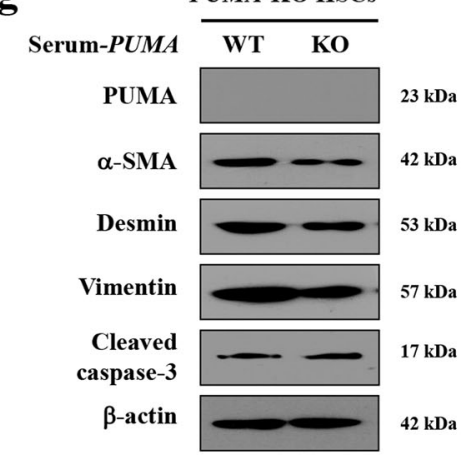

Fig. 9 NF-KBp65/PUMA-driven liver inflammation-induced HSCs activation and liver fibrosis. a Schematic diagram of the serum test in vitro. $\mathbf{b}$ The genes expressions of the indicated inflammatory cytokines from $\mathrm{CCl}_{4}$-treated PUMA-WT or PUMA-KO mice were detected by quantitative reversetranscription PCR. The expression of $\beta$-actin in each tissue was quantified as the internal control. $n=6$ per group. ${ }^{*} P<0.05$. c Representative images of the growth with activation of primary isolated $\mathrm{HSCS}$ following the treatment of the distinct serum extracted from $\mathrm{CCl}_{4}$-treated PUMA-WT or PUMA$\mathrm{KO}$ mice. Nuclei (blue) were counterstained with DAPI. $\mathbf{d}$ Expressions of the related proteins of primary HSCs after serum treatment were detected by western blotting, revealing that PUMA-WT serum enhanced the activation of HSCs without affecting cell apoptosis. $n=6$ per group. e Schematic diagram of the serum test in vivo. $\mathbf{f}$ Sirius red staining (red) and a-SMA staining (red) were examined in the liver tissues of PUMA-KO mice following the treatment of the serum extracted either from $\mathrm{CCl}_{4}$-treated PUMA-WT or PUMA-KO mice. The primary isolated HSCs were also analyzed by a-SMA staining (red). Nuclei (blue) were counterstained with DAPI. $\mathbf{g}$ The indicated proteins of the primary isolated HSCs from PUMA-KO mice after serum treatment were detected by western blotting, $n=6$ per group. 
revealed that deletion of $N F-\kappa B p 65$ in hepatocytes enhanced the expressions of collagen-IV, collagen-I, and $\alpha$-SMA, along with the increased number of apoptotic hepatocytes in mice, which finally aggravated liver fibrosis. During this process, downregulated or absent NFкBp65 activity in hepatocytes, which leads to increased hepatocytes apoptosis, plays an important role in the initiation of liver fibrosis. We then examined the Bcl-2 family, which integrates a number of inter- and intracellular cues to determine whether or not the apoptosis pathway should be activated, and found that the prosurvival Bcl-2 family members were downregulated in hepatocytes with specific deletion of $N F-\kappa B p 65$, while the potent pro-apoptotic element PUMA was increased following NF-KBp65 deficiency in hepatocytes, which contributed to the development of liver fibrosis. By the way, the hepatic fibrosis mouse model was established by $\mathrm{CCl}_{4}$ for 4 weeks and 8 weeks, and we found that the mouse obtained the highest apoptosis index at the 4th week, and then the apoptotic cells decreased gradually as time goes on. During this process, liver fibrosis was accelerated progressively by the accumulation of collagen and the formation of septa, with a maximum peak at 8th week. This phenomenon may be related to that hepatocyte apoptosis is a pivotal and initial step in the development of liver fibrosis, and the pathogenesis of liver fibrosis owns different pathophysiological changes (the changes of hepatocyte apoptosis, collagen deposition, etc) in the different stage ${ }^{6,7,21}$, and our further study is needed to determine the definite causes of this phenomenon.

Previous studies have recognized that dying hepatocytes release alarmins that could recruit inflammatory cells to produce inflammatory cytokines to regulate the initiation and development of liver fibrosis ${ }^{8}$. In our study, inflammatory cells and mediators were dramatically upregulated in NF-kBp65 deletion in hepatocytes in liver fibrosis, while PUMA antisense oligonucleotides or PUMA deficiency suppressed that inflammatory response. Some researches have shown that the mechanisms by which apoptosis promotes inflammation relate to the activation of the resident macrophages in the liver, and HSCs undergo a process of activation resulting from hepatocytes apoptosis and inflammation $^{4,49,50}$. Adopting the TGF- $\beta 1$ inhibitor pirfenidone, TNF- $\alpha$ inhibitor pentoxifylline, and monocytes depressor clodronate-loaded liposomes, we demonstrated that inhibition of inflammatory action ameliorated HSCs activation and liver fibrosis. Moreover, our in vivo and in vitro serum data suggested hepatocytes apoptosis contributed to inflammation-induced HSCs activation. These results indicated that HSCs activation depended on the inflammatory response following hepatocytes apoptosis. However, there is much yet to be learned about the complex interplay between the immune system and the hepatocytes status in the pathophysiological states of the liver.
In summary, our investigation provides evidence that Fas/FasL contributes to NF-kBp65/PUMA-regulated hepatocytes apoptosis via autophagy to enhance HSCs activation and liver fibrosis, and this network could be a therapeutic target for liver fibrosis.

\section{Materials and methods \\ Tissue samples}

Six normal liver tissues were from parahemangioma sites of hepatic hemangioma patients and the paired samples of liver fibrosis were obtained from 6 hepatitis B virus (HBV)-infected liver fibrosis patients during operations before any therapeutic intervention. Written informed consent was received from each patient and healthy volunteer prior to inclusion in the study and the acquisition of these samples was approved by the Clinical Research Ethics Committee of the Third Affiliated Hospital of Sun Yat-Sen University.

\section{Mice and treatments}

All animal experiments were approved by the Institutional Animal Ethics Committee of the Third Affiliated Hospital of Sun Yat-Sen University. Eight- to ten-weekold male mice (20-25 g) were used for all experiments. All mice were randomly allocated to each group and to collect and process data for analysis, and the experimenters were blinded towards the treatments or genetic background in all the experiments. LoxP NF- KBp65 (RelA) mice were generated on a C57BL/6 gene background. Hepatocytes specific $N F-\kappa B p 65$ deletion mice ( $p 65 \Delta$ hepa) were generated by crossing the floxed $p 65$ mice with $A l b$ cre mice, which shows hepatocyte-specific expression of Cre recombinase and NF- $\mathrm{KBp} 65$ ablated solely in hepatocytes but not in non-parenchymal liver cells. Floxed $p 65$ littermates $\left(p 65^{f / f}\right)$ were used as the wild-type (WT) mice. PUMA wild type (PUMA-WT) and PUMA knockout (PUMA-KO) littermates on a C57BL/6 background were generated from PUMA heterozygote mice (Jackson Laboratory, Bar Harbor, ME, USA). p $65^{f / f} / P U M A-W T$ mice on a C57BL/6 background generated by crossing p $65^{f / f}$ and PUMA-WT littermates, p65 2 hepa/PUMA-WT mice on a C57BL/6 background were generated from p65 $\triangle$ hepa and PUMA-WT littermates.

The $\mathrm{CCl}_{4}$-induced liver fibrosis model was established via an intraperitoneal injection of $20 \% \mathrm{CCl}_{4}$ (Sinopharm Chemical Reagent, Shanghai, China) dissolved in an olive oil solution (Sinopharm Chemical Reagent) at $5 \mathrm{ml} / \mathrm{kg}$ body weight, twice per week for 8 weeks. The control group was intraperitoneally injected only with olive oil at $5 \mathrm{ml} / \mathrm{kg}$ body weight, twice per week for 8 weeks. For inhibition of autophagy, the autophagic inhibitor 3-methyladenine (3MA, Sigma, St Louis, MO, USA) was given with $3 \mu \mathrm{l}$ of a $20 \mathrm{mg} / \mathrm{ml}$ solution prepared in saline, and mice were injected with $3-\mathrm{MA}(10 \mathrm{mg} / \mathrm{kg})$ at $30 \mathrm{~min}$ before every $\mathrm{CCl}_{4}$ 
injection, and the vehicle group (as Vehicle) was injected with the same volume of saline. For inhibiting inflammatory response, mice were orally administrated with TGF- $\beta 1$ inhibitor pirfenidone (Sigma) at $250 \mathrm{mg} / \mathrm{kg}$ daily for 8 weeks. To inhibit TNF- $\alpha$ action, pentoxifylline (Sigma) at $200 \mathrm{mg} / \mathrm{kg}$ was administered by intraperitoneal injection for 8 weeks to mice at $30 \mathrm{~min}$ before every $\mathrm{CCl}_{4}$ injection. Clodronate-loaded liposomes (Sigma) were intraperitoneally injected in order to deplete monocytes for three consecutive days per week for 8 weeks in the process of mice models. To inhibited PUMA expression in mice, the PUMA sense oligonucleotides ( $5^{\prime}-\mathrm{A}^{*} \mathrm{G}^{*} \mathrm{C}$ "GCCATGGCCC GCGC*A*C*G-3'; "phosphorothioate bonds) and PUMA antisense oligonucleotides (PUMA-AS, $5^{\prime}-\mathrm{C}^{*} \mathrm{G}^{*} \mathrm{~T} * \mathrm{GCGCG}$ GGCCATGGC* $\left.\mathrm{G}^{*} \mathrm{C}^{*} \mathrm{~T}-3^{\prime}\right)$ were synthesized by GenePharma (Shanghai, China). The mice were treated with PUMA sense or PUMA antisense oligo at $25 \mathrm{mg} / \mathrm{kg} /$ day via intraperitoneal injection for 8 weeks. By inhibiting the $\mathrm{Bcl}-2$ activity, ABT-199 (Venetoclax, $100 \mathrm{mg} / \mathrm{kg} / \mathrm{d}$, Santa Cruz, Santa Cruz, CA, USA) was utilized via oral gavage. Six animals were used in each group for the above studies.

\section{Primary cells isolation and cells culture}

Primary hepatocytes and HSCs were dissociated from the indicated mice by a non-recirculating collagenase perfusion as previously described ${ }^{21,51}$. Liver was perfused through the portal vein in situ successively with $\mathrm{Ca}^{2+}$-free HBSS (Hank's Balanced Salt solution) for $15 \mathrm{~min}$, with $100 \mathrm{ml} 0.2 \%$ pronase solution, and lastly with $0.2 \%$ collagenase Type-IV (Sigma) solution until the liver looked digested and became pale in color. This resulting cell suspension was filtered through a $100 \mu \mathrm{m}$ pore size mesh nylon filter (Sinopharm Chemical Reagent), and then centrifuged for $10 \mathrm{~min}$ at $200 \times g$, the pellet was collected for primary hepatocytes and the supernate for primary HSCs. The primary hepatocytes were cultured in RPMI medium 1640 supplemented with 10\% heat-inactivated fetal bovine serum (FBS), 100 units $/ \mathrm{ml}$ penicillin, and $100 \mathrm{\mu g} / \mathrm{ml}$ streptomycin in a humidified incubator at $37^{\circ} \mathrm{C}$ with $5 \% \mathrm{CO}_{2}$. For HSCs isolation, commercially available $60 \%$ Optiprep (Sigma) was added to the final concentration $11.5 \%$. The above supernate was centrifuged at $450 \times$ $\mathrm{g}$ for $15 \mathrm{~min}$ and then suspended with $0.5 \mathrm{ml} \mathrm{HBSS}$, after being centrifuged at $1400 \times g$ for $25 \mathrm{~min}$, HSCs on the top of Optiprep layer were collected and also cultured in RPMI medium 1640. These cells were authenticated and tested and they were not contaminated by mycoplasma. For FasL treatment experiments in vitro, FasL $(10 \mathrm{ng} / \mathrm{ml}$, Sigma) was added for $12 \mathrm{~h}$, and siRNA treatment was performed as previously described ${ }^{29}$.

\section{Serum test}

For serum test in vitro, following induction of liver fibrosis in $\mathrm{CCl}_{4}$-induced PUMA-WT and PUMA-KO mouse models, these mice were anesthetized and their serum was extracted, respectively. The above-mentioned different serum was added to the primary HSCs isolated from normal PUMA-WT mice (without any treatments), respectively, and then cultured in a humidified incubator at $37{ }^{\circ} \mathrm{C}$ with $5 \% \mathrm{CO}_{2}$ for $36 \mathrm{~h}$. For serum test in vivo, the serum was extracted from $\mathrm{CCl}_{4}$-induced liver fibrosis models in PUMA-WT and PUMA-KO mice, respectively. The above-mentioned distinct serum was then transfused to the different PUMA-KO littermates (by the third week of $10 \% \mathrm{CCl}_{4}$ treatment, at $200 \mu \mathrm{l}$ per mouse for three consecutive days per week until the 8th week) via tail intravenous injection. Following that, the mice were anesthetized and euthanized and the entire liver was analyzed using histopathological detection.

\section{Histological staining}

Sirius red staining was used for collagen determination. Hematoxylin-esoin (H\&E) staining, immunohistochemical (IHC), immunofluorescence (IF), and double IF staining were also performed as previously described ${ }^{51}$, and the semi-quantitative analysis of the histological staining and sirius red staining was analyzed using ImagePro Plus 6.0. IHC and IF staining were performed by using antibodies for Fas (SAB5700608), FasL (SAB4501538), NF-кBp-p65 (p-p65, SAB4504482), NF-кBp65 (p65, SAB4502615) (all from Sigma), collagen-I (COL-I, ab233080), $\alpha$-SMA (ab5694), vimentin (ab92547), PUMA (ab9643), transferrin (ab278498), TGF- $\beta$ (ab215715), MPO (ab208670) (all from Abcam, Cambridge, MA, USA), desmin (sc-23879), BECN1 (sc-48341), IL-6 (sc28343), F4/80 (sc-52664) (all from Santa Cruz), cleaved caspase-3 (9661), CD3 (86603), TNF- $\alpha$ (11948), PCNA (13110) (all from Cell Signaling Technology, Danvers, MA, USA). TUNEL staining was performed using the In Situ Cell Death Detection Kit (Roche, Basel, Switzerland) according to the manufacturer's instructions. The apoptotic index was determined by dividing the number of apoptotic cells by the total number of cells in the section in at least 10 randomly selected fields $(\times 200)$. For transmission electron microscopy, the hepatic tissues were fixed as previously described ${ }^{21}$ and observed with a transmission electron microscope (Hitachi, H-800, Tokyo, Japan), and the images were acquired digitally from a randomly selected pool of six fields.

\section{Western blotting}

The related proteins were analyzed by western blotting using anti- $\alpha$-SMA (ab5694), -vimentin (ab92547), -PUMA (ab9643), -collagen-IV (COL-IV, ab236640, ab6586), -COL-I (ab233080) (all from Abcam), -Fas (SAB5700608), -FasL (SAB4501538), -NF-kBp-p65 (p-p65, SAB4504482), -NFкBp65 (p65, SAB4502615), - $\beta$-actin (A5441) (all from Sigma), -desmin (sc-23879), -Bcl-xL (sc-8392), -Mcl-1 (sc-74437), 
-Bcl-2 (sc-7382), -BECN1 (sc-48341) (all from Santa Cruz) and -cleaved caspase-3 (9661) (Cell Signaling Technology). Appropriate horseradish peroxidase conjugated secondary antibodies were used to detect the primary antibody/antigen complexes as previously described ${ }^{35}$, and then was quantified for densitometry analysis. For controlling unwanted sources of variation, the quantitative densitometry results were calculated and normalized to the loading control $\beta$-actin densitometry units.

\section{RNA extraction and PCR assays}

Total RNA was extracted using the RNAgents Total RNA Isolation System (Promega, Madison, WI, USA) according to the manufacturer's instruction. Real-time polymerase chain reaction (PCR) was performed on a Chromo 4 Detector System (MJ Research, Sierra Point, CA, USA) using gene-specific primers and DyNAmo SYBR Green Master Mix (Finnzymes, Finland). Relative mRNA ratio was used to analyze the data, and the indicated mRNA in each tissue was normalized by $\beta$-actin gene (as the internal control) from the same tissue and expressed as fold changes relative to the matched control values.

\section{Microarray experiment}

Primary hepatocytes were dissociated from three normal liver samples with olive oil treatment and three matched pairs of fibrotic tissues with $\mathrm{CCl}_{4}$ treatment, respectively. Total RNA was isolated by using TRIzol reagent (Invitrogen, Carlsbad, CA, USA), and the total RNA was amplified, labeled, and purified by Affymetrix WT PLUS Reagent Kit (Affymetrix, Santa Clara, CA, USA) and FL-Ovation cDNA Biotin Module V2 (NuGEN, San Carlos, CA, USA) to obtain the biotinlabeled cDNA. Array hybridization and washing were performed using GeneChip Hybridization, Wash and Stain Kit (Affymetrix) in a Hybridization Oven 645 (Affymetrix) and a Fluidics Station 450, and then the arrays were scanned by Affymetrix GeneChip Scanner 3000 (Affymetrix). Command Console Software (Affymetrix) was used to control the scanner and summarize probe cell intensity data (CEL file generation) with default settings. The array data were analyzed for data summarization, normalization, and quality control using the GeneSpring software V12 (Agilent). The data were Log2 transformed and median centered by genes using the Adjust Data function of CLUSTER 3.0 software and then further analyzed with hierarchical clustering with average linkage. Finally, tree visualization was performed by using Java Treeview (Stanford University School of Medicine, Stanford, CA, USA). The microarray dataset is available in the figshare repository (https://doi.org/10.6084/m9.figshare.14443382).

\section{Statistical analysis}

The experimenters were blinded towards the treatments or genetic background in all the experiments. To ensure adequate power to detect a pre-specified effect, the sample size was chosen using the Power Analysis and Sample Size (PASS) software. The sample size was estimated even if no statistical methods were used in animal studies. At least six mice or human sections were adopted in each group. Statistical analysis was performed only for studies where each group size was at least $n=6$, unless otherwise stated. These data were normal distribution and were presented as mean \pm SEM, and data statistical analysis were performed using Student's two-tailed paired $t$-test or one-way ANOVA (more than two groups of data, single factor) or two-way ANOVA (more than two groups of data, two factors), followed by Bonferroni's comparison post hoc test and post hoc tests are run only if $F$ achieved $P<0.05$. There was no significant variance inhomogeneity, and the variance was similar between the groups that were being statistically compared. Differences were considered statistically significant at the level of $P<0.05$.

\section{Acknowledgements}

We would like to acknowledge the reviewers for their helpful comments on this paper.

\section{Author contributions \\ S.W.T. and X.Z.L. performed the experiments, analyzed the data, and revised the manuscript; L.J.C. and X.Q.W. collected the clinical samples and conducted the clinical study; L.T. and X.M.P. contributed the essential reagents and conducted the animals' study; S.Y.T. and H.L.L. planned and conducted primary cells isolation; J.J. performed the cells culture study; B.W. designed the whole project, supervised the research, wrote the paper and approved of the final version.}

\section{Funding}

This work was supported by grants from the Science and Technology Planning Projects of Guangzhou City (201804010026), the major talent project training program of the Third Affiliated Hospital of Sun Yat-Sen University (P02089), the National Natural Science Foundation of China $(81700536$, 82070574), the Natural Science Foundation of Guangdong Province (2017A030310188), the Basic Research Programme of Young Teachers' Training Project of Sun Yat-sen University (17ykpy51).

\section{Data availability}

All data generated or analyzed during this study are included in this published article [and its supplementary information files].

\section{Ethics statement}

Written informed consent was received from each patient and healthy volunteer prior to inclusion in the study and the research process was approved by the Clinical Research Ethics Committee of the Third Affiliated Hospital of Sun Yat-Sen University. All animal experiments were approved by the Institutional Animal Ethics Committee of the Third Affiliated Hospital of Sun Yat-Sen University.

\section{Conflict of interest}

The authors declare no competing interests. 


\section{Publisher's note}

Springer Nature remains neutral with regard to jurisdictional claims in published maps and institutional affiliations.

Supplementary information The online version contains supplementary material available at https://doi.org/10.1038/s41419-021-03749-x.

Received: 30 January 2021 Revised: 15 April 2021 Accepted: 19 April 2021 Published online: 12 May 2021

\section{References}

1. Lee, Y. A., Wallace, M. C. \& Friedman, S. L. Pathobiology of liver fibrosis: a translational success story. Gut 64, 830-841 (2015).

2. Yin, C., Evason, K. J., Asahina, K. \& Stainier, D. Y. Hepatic stellate cells in liver development, regeneration, and cancer. J. Clin. Invest. 123, 1902-1910 (2013).

3. Pradere, J. P. et al. Hepatic macrophages but not dendritic cells contribute to liver fibrosis by promoting the survival of activated hepatic stellate cells in mice. Hepatology 58, 1461-1473 (2013).

4. Suh, Y. G. et al. CD11b(+) Gr1(+) bone marrow cells ameliorate liver fibrosis by producing interleukin-10 in mice. Hepatology 56, 1902-1912 (2012).

5. Kornek, M., Popov, Y., Libermann, T. A., Afdhal, N. H. \& Schuppan, D. Human T cell microparticles circulate in blood of hepatitis patients and induce fibrolytic activation of hepatic stellate cells. Hepatology 53, 230-242 (2011).

6. Qian, H. et al. An HNF1alpha-regulated feedback circuit modulates hepatic fibrogenesis via the crosstalk between hepatocytes and hepatic stellate cells. Cell Res. 25, 930-945 (2015).

7. Jung, $Y$. et al. Signals from dying hepatocytes trigger growth of liver progenitors. Gut 59, 655-665 (2010).

8. Chung, S. I. et al. Hepatic expression of Sonic Hedgehog induces liver fibrosis and promotes hepatocarcinogenesis in a transgenic mouse model. J. Hepatol. 64, 618-627 (2016).

9. Luedde, T. \& Schwabe, R. F. NF-kappaB in the liver-linking injury, fibrosis and hepatocellular carcinoma. Nat. Rev. Gastroenterol. Hepatol. 8, 108-118 (2011).

10. Elliott, M. R. et al. Nucleotides released by apoptotic cells act as a find-me signal to promote phagocytic clearance. Nature 461, 282-286 (2009).

11. Guicciardi, M. E. \& Gores, G. J. Life and death by death receptors. Faseb J. 23 1625-1637 (2009).

12. Kotov, D. I., Kotov, J. A., Goldberg, M. F. \& Jenkins, M. K. Many Th cell subsets have Fas ligand-dependent cytotoxic potential. J. Immunol. 200, 2004-2012 (2018).

13. Qadir, A. S. et al. CD95/Fas Increases stemness in cancer cells by inducing a STAT1-dependent Type I interferon response. Cell Rep. 18, 2373-2386 (2017).

14. Wang, K. Autophagy and apoptosis in liver injury. Cell Cycle 14, 1631-1642 (2015).

15. Li, B. et al. Cell apoptosis and Fas gene expression in liver and renal tissues after ischemia-reperfusion injury in liver transplantation. Transpl. Proc. 42, 1550-1556 (2010).

16. Reinehr, R. \& Häussinger, D. CD95 death receptor and epidermal growth factor receptor (EGFR) in liver cell apoptosis and regeneration. Arch. Biochem. Biophys. 518, 2-7 (2012).

17. Magraoui, F. E., Reidick, C., Meyer, H. E. \& Platta, H. W. Autophagy-related deubiquitinating enzymes involved in health and disease. Cells-Basel $\mathbf{4}$, 596-621 (2015).

18. Shaffer, L. Out with the bad: studying autophagy to fight infectious disease. Nat. Med. 22, 334-335 (2016).

19. Sridhar, S., Botbol, Y., Macian, F. \& Cuervo, A. M. Autophagy and disease: always two sides to a problem. J. Pathol. 226, 255-273 (2012).

20. Czaja, M. J. et al. Functions of autophagy in normal and diseased liver. Autophagy 9, 1131-1158 (2013).

21. Tan, S. et al. beta-Arrestin1 enhances liver fibrosis through autophagymediated Snail signaling. Faseb J. 33, 2000-2016 (2019).

22. Czerkies, M. et al. Cell fate in antiviral response arises in the crosstalk of IRF, NFkappaB and JAK/STAT pathways. Nat. Commun. 9, 493 (2018).

23. Li, Y. et al. NS5ATP13 promotes liver fibrogenesis via activation of hepatic stellate cells. J. Cell Biochem. 118, 2463-2473 (2017).

24. Feng, G. S. Conflicting roles of molecules in hepatocarcinogenesis: paradigm or paradox. Cancer Cell 21, 150-154 (2012).
25. Shen, $\mathrm{H}$. et al. Thymic NF-kappaB-inducing kinase regulates CD4+ T cellelicited liver injury and fibrosis in mice. J. Hepatol. 67, 100-109 (2017).

26. Koppe, C. et al. IkappaB kinasealpha/beta control biliary homeostasis and hepatocarcinogenesis in mice by phosphorylating the cell-death mediator receptor-interacting protein kinase 1. Hepatology 64, 1217-1231 (2016).

27. Schuppan, D. \& Kim, Y. O. Evolving therapies for liver fibrosis. J. Clin. Invest. 123, 1887-1901 (2013).

28. Delbridge, A. R., Opferman, J. T., Grabow, S. \& Strasser, A. Antagonism between MCL-1 and PUMA governs stem/progenitor cell survival during hematopoietic recovery from stress. Blood 125, 3273-3280 (2015).

29. Tan, S. et al. beta-Arrestin-1 protects against endoplasmic reticulum stress/p53upregulated modulator of apoptosis-mediated apoptosis via repressing $\mathrm{p}$ p65/inducible nitric oxide synthase in portal hypertensive gastropathy. Free Radic. Biol. Med. 87, 69-83 (2015).

30. Sun, Q. et al. Proapoptotic PUMA targets stem-like breast cancer cells to suppress metastasis. J. Clin. Invest. 128, 531-544 (2018).

31. Yu, J. \& Zhang, L. PUMA, a potent killer with or without p53. Oncogene 27, S71-S83 (2008).

32. Li, Y. et al. The p53-PUMA axis suppresses iPSC generation. Nat. Commun. 4 2174 (2013).

33. Weber, A. et al. Hepatocyte-specific deletion of the antiapoptotic protein myeloid cell leukemia-1 triggers proliferation and hepatocarcinogenesis in mice. Hepatology 51, 1226-1236 (2010).

34. Wang, P. et al. PUMA is directly activated by NF-kappaB and contributes to TNF-alpha-induced apoptosis. Cell Death Differ. 16, 1192-1102 (2009).

35. Tan, S. et al. IL-6-driven FasL promotes NF-kappaBp65/PUMA-mediated apoptosis in portal hypertensive gastropathy. Cell Death Dis. 10, 748 (2019)

36. Pihán, P., Carreras-Sureda, A. \& Hetz, C. BCL-2 family: integrating stress responses at the ER to control cell demise. Cell Death Differ. 24, 1478-1487 (2017).

37. Chipuk, J. E. \& Green, D. R. PUMA cooperates with direct activator proteins to promote mitochondrial outer membrane permeabilization and apoptosis. Cell Cycle 8, 2692-2696 (2009).

38. Ren, D. et al. BID, BIM, and PUMA are essential for activation of the BAX- and BAK-dependent cell death program. Science 330, 1390-1393 (2010).

39. Wu, B. et al. Downregulation of cyclooxygenase-1 is involved in gastric mucosal apoptosis via death signaling in portal hypertensive rats. Cell Res. 19, 1269-1278 (2009).

40. Xiong, S., Mu, T., Wang, G. \& Jiang, X. Mitochondria-mediated apoptosis in mammals. Protein Cell 5, 737-749 (2014).

41. Park, M. A. et al. Sorafenib activates CD95 and promotes autophagy and cell death via Src family kinases in gastrointestinal tumor cells. Mol. Cancer Ther. 9, 2220-2231 (2010)

42. de Giorgio, R. et al. Neurogenic chronic intestinal pseudo-obstruction: antineuronal antibody-mediated activation of autophagy via Fas. Gastroenterology 135, 601-609 (2008).

43. Zhang, Y. et al. Fas-mediated autophagy requires JNK activation in HeLa cells. Biochem. Biophys. Res. Commun. 377, 1205-1210 (2008).

44. Xie, W. et al. Chaperone-mediated autophagy prevents apoptosis by degrading BBC3/PUMA. Autophagy 11, 1623-1635 (2015).

45. Thorburn, J. et al. Autophagy controls the kinetics and extent of mitochondrial apoptosis by regulating PUMA levels. Cell Rep. 7, 45-52 (2014).

46. Tan, S. et al. PUMA mediates ER stress-induced apoptosis in portal hypertensive gastropathy. Cell Death Dis. 5, e1128 (2014).

47. Hoesel, B. \& Schmid, J. A. The complexity of NF-kappaB signaling in inflammation and cancer. Mol. Cancer 12, 86 (2013).

48. Huang, $X$. et al. miR-196b-5p-mediated downregulation of FAS promotes NSCLC progression by activating IL6-STAT3 signaling. Cell Death Dis. 11, 785 (2020).

49. Bárcena, C. et al. Gas6/Axl pathway is activated in chronic liver disease and its targeting reduces fibrosis via hepatic stellate cell inactivation. J. Hepatol. 63, 670-678 (2015).

50. Karsdal, M. A. et al. Novel insights into the function and dynamics of extracellular matrix in liver fibrosis. Am. J. Physiol. Gastrointest. Liver Physiol. 308, G807-G830 (2015).

51. Tan, S., Liu, H., Ke, B., Jiang, J. \& Wu, B. The peripheral CB1 receptor antagonist JD5037 attenuates liver fibrosis via a CB1 receptor/beta-arrestin1/Akt pathway. Br. J. Pharm. 177, 2830-2847 (2020). 